Document downloaded from:

http://hdl.handle.net/10251/142954

This paper must be cited as:

Sharma, S.; Broatch, A.; Garcia Tiscar, J.; Nickson, AK.; Allport, JM. (05-2). Acoustic and pressure characteristics of a ported shroud turbocompressor operating at near surge conditions. Applied Acoustics. 148:434-447. https://doi.org/10.1016/j.apacoust.2019.01.005

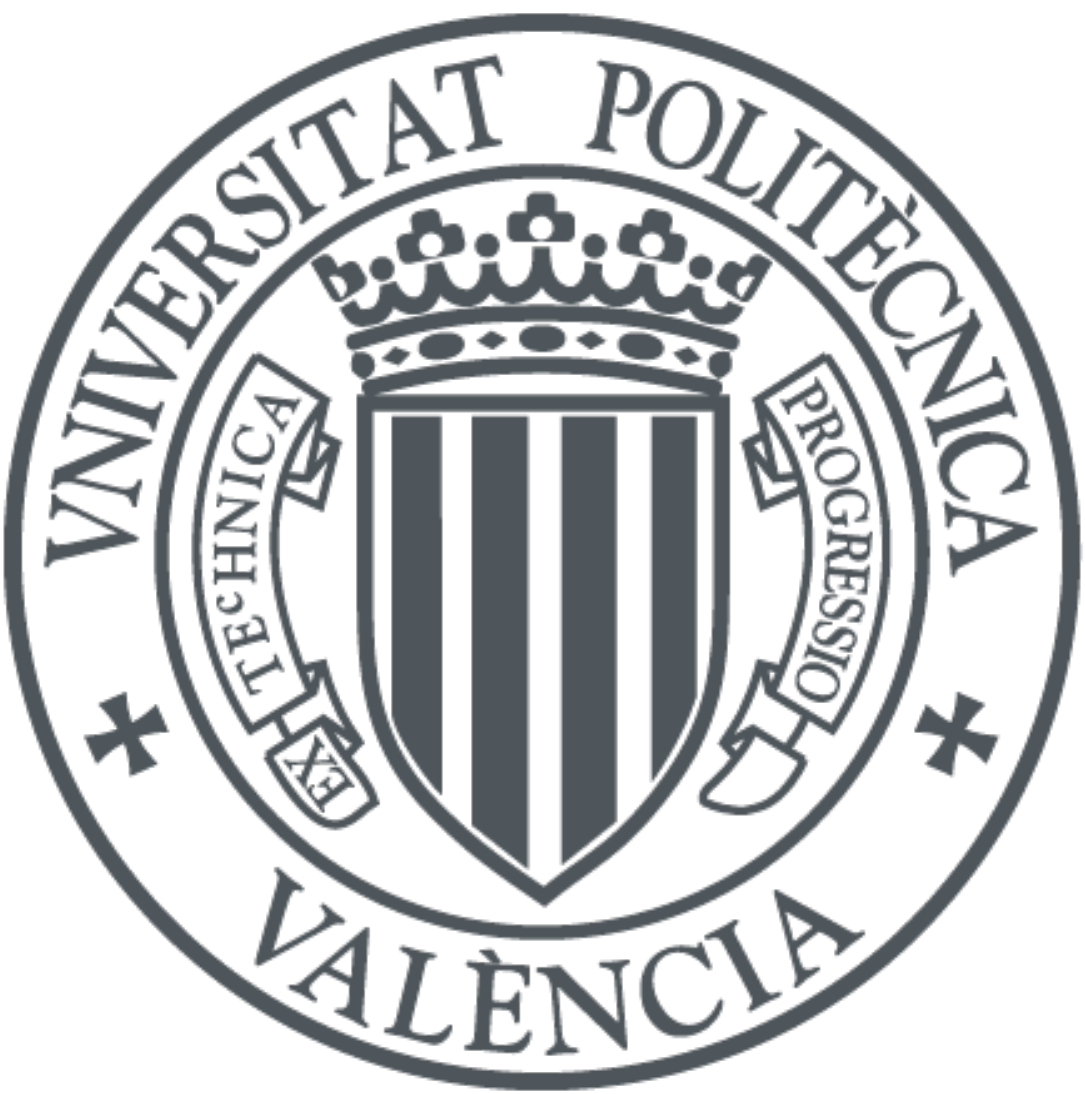

The final publication is available at

https://doi.org/10.1016/j.apacoust.2019.01.005

Copyright Elsevier

Additional Information 


\title{
Acoustic and pressure characteristics of a ported shroud turbocompressor operating at near surge conditions
}

\author{
S. Sharma ${ }^{\mathrm{a}, *}$, A. Broatch ${ }^{\mathrm{b}}$, J. García-Tíscar ${ }^{\mathrm{b}}$, A. K. Nickson ${ }^{\mathrm{c}}$, J. M. Allport ${ }^{\mathrm{a}}$ \\ ${ }^{a}$ Turbocharger Research Institute, University of Huddersfield, Huddersfield, HDI 3DH, United Kingdom \\ ${ }^{b}$ CMT - Motores Térmicos, Universitat Politècnica de València, Camino de Vera, 46022 Valencia, Spain \\ ${ }^{c}$ BorgWarner Turbo Systems Division, Bradford, West Yorkshire BD4 6SE, United Kingdom
}

\begin{abstract}
The operation of compressor at lower mass flow rates is limited by surge which is marked by large fluctuations in operational variables and accompanied by significant increase in noise. Ported shroud casing treatment is a widely used method to control the flow near unstable conditions in order to obtain a stable operation and enhance deep surge margin. The research on the acoustic effects of the ported shroud design is limited. Therefore, this paper numerically characterises the acoustic features of a turbocharger compressor with ported shroud design operating at marginal or soft surge conditions and investigates the correlation between acoustic characteristics and the spatial flow structures. The acoustic and the flow field features are analysed using spectral signatures obtained from an experimentally validated numerical model using both performance and acoustic measurements. Propagation of the frequency content through the ducts has been estimated with the aid of the beamforming and method of characteristics to enhance the content coming from the compressor. Expected acoustic phenomena such as rotating order tones and blade passing peaks are correctly identified in the modelled spectrum with the limitation to capture the specific broadband features. Hence, the numerical model can be used to further the research encompassing the impact these flow enhancement solutions have on the noise emission of the turbocharger. Inspection of the flow field shows radially exiting fluid at the ported shroud slot leading to the formation of the high-speed jets exiting the ported shroud cavity. Circumferential propagation of the stall cells is also identified in the impeller. Further inspection of the pressure field through modal decomposition implies the localisation of the energetic noise sources in the impeller downstream components. The influence of the ported shroud cavity on the acoustic characteristic of the compressor is not significant and is limited to the propagation of the tonal noise in the direction of impeller upstream.
\end{abstract}

Keywords: LES, Compressor, Ported Shroud, Surge, Noise, Modal Decomposition

\section{Introduction}

Surge corresponds to a system instability effect which manifests as strong fluctuations in various aerodynamic and thermodynamic parameters such as pressure, temperature, mass flow rate and noise. The state of surge challenges the functionality and structural integrity of the system and therefore, limits the operation of the turbomachine. The surge in compressors is seen for the operating region of lower mass flow rates and is characterised by a positive inflexion in the pressure ratio curve. Surge is subjectively classified as hard or deep surge and mild or marginal or soft surge based on the amplitude of the fluctuations of aerothermodynamic varriables. Mild or soft surge is marked by the oscillatory behaviour of the system variables that usually form a series of successive stable and unstable states [1], while deep or hard surge is a catastrophically violent, inherently unstable state. Both mild and deep surge are known for significantly increasing the acoustic levels. A distinct increase in the magnitude of flow instabilities is noticed prior to the onset of the surge. The weight of literature points towards local flow

${ }^{*}$ Corresponding author. Tel.: +44 7920074808 , email: sidharath.sharma@hud.ac.uk instabilities that arise due to the separated flow caused by the increased angle of incidence as the mass flow rate is decreased at the constant operating speed. These local instabilities tend to stall the impeller blade and are known to propagate circumferentially at a speed lower than that of the impeller. The flow instabilities (stall) can either develop or propagate to the other components of the compressor i.e. diffuser and inducer. The local flow instabilities may or may not lead the system to surge. However, local degradation of the flow is believed to create conditions that eventually lead to the surge.

The onset of surge can be delayed by using several active and passive flow control methods which alleviates the impact of these local flow instabilities introduced at lower mass flow rates. Casing treatment, specifically Ported Shroud (PS) [2, 3] casing treatment is one such widely used passive method to increase the operating range in turbocharger compressors. The low momentum fluid that blocks the blade passage is recirculated back to the compressor inlet using ported shroud design for achieving the stability near surge. Although performances deterioration associated with the ported shroud casing treatment are well established, little is studied in terms of their impact on acoustic characteristics of the compressor.

The acoustic emission of the compressor at the off-design 


\section{Nomenclature}

$a$ speed of sound $\left(\mathrm{ms}^{-1}\right)$

$\mathbf{a}_{i}$ Time evolution of POD mode $i$

$D$ duct diameter (m)

$D_{e}$ exducer diameter (m)

$f$ frequency $(\mathrm{Hz})$

$f_{c, a}$ First asymmetric mode frequency $(\mathrm{Hz})$

$f_{c}$ Nyquist cut-off frequency $(\mathrm{Hz})$

$L$ sensor separation (m)

$\dot{m}$ mass flow rate $\left(\mathrm{kgs}^{-1}\right)$

$M$ Mach number (-)

$t$ time (s)

$T$ temperature (K)

$T_{\text {out }, 0}$ total temperature at the outlet boundary (K)

$u$ axial velocity $\left(\mathrm{ms}^{-1}\right)$

$\dot{W}$ compressor absorbed work $\left(\mathrm{kgm}^{2} \mathrm{~s}^{-3}\right)$

$\varepsilon$ relative error (\%)

$\gamma$ ratio of specific heats $(-)$

$\phi$ generic variable

$p_{\text {out }, 0}$ total pressure at the outlet boundary $(\mathrm{Pa})$

$\boldsymbol{\Psi}_{i}$ spatial distribution of POD mode $i$

$\Pi_{\mathrm{t}-\mathrm{t}}$ total-to-total pressure ratio (-)

$\eta_{s}$ isentropic efficiency (-) $y^{+}$dimensionless wall distance (-)

$\mathbf{V}_{1}^{N}$ matrix of pressure snapshots $\mathbf{v}_{i}, i \in[1, \ldots, N]$

BPF blade passing frequency $(\mathrm{Hz})$

CFD computational fluid dynamics

DES detached eddy simulation

IBC impeller backside cavity

LCMV linearly-constrained minimum variance

LES large eddy simulation

$\mathrm{NVH}$ noise vibration harshness

PIV particle-imaging velocimetry

POD proper orthogonal decomposition

PS ported shroud

PSD pressure spectral density $(\mathrm{dB})$

RANS Reynolds-averaged Navier-Stokes

$\mathrm{RBM}$ rigid body motion

$\mathrm{RO}$ rotation order (-)

RSI rotor-stator interaction

SBES stress-blended eddy simulation

SST shear-stress transport

TCN tip clearance noise

WALE wall adopted local eddy viscosity and near surge condition is an area of active research, specifically by automotive and turbocharger manufactures due to the customer sensitivity to noise. The acoustic spectrum of the compressor operating near surge is characterised specifically by broadband noise identified with the onomatopoeia like 'whoosh' [4, 5] or 'hiss' [6] or 'blow' [7] noise. Literature shows varied values when it comes to frequency range of the 'whoosh' noise ranging from $1.5-3 \mathrm{kHz}[7,5]$ to $4-12 \mathrm{kHz}[8,4]$. Torregrosa et al. [9] points towards the conflation with the so called Tip Clearance Noise (TCN) by the authors $[8,4]$ identifying an inclusive range of 'whoosh' noise. Earlier studies [4, 5] identified 'whoosh' noise as a specific near surge or marginal surge phenomenon but recent literature $[10,11]$ shows the presence of broadband noise at design conditions too. Raitor and Neise [12] measured the acoustic spectra of the centrifugal compressor at various speedlines but near surge operation was not studied. Gaude et al. [6] presented an exhaustive characterisation of turbocharger noise. Although acoustic investigations in literature are predominately of experimental nature, the work of Mendonca et al. [13] established the potential of computational fluid dynamics to model the compressor acoustics. Furthemore, Broatch et al. [14] presented reasonable correlation of modelled acoustic spectrum with the experimentally measured data.

Recent numerical work presented by the researchers of KTH $[15,16,17]$ investigated the performance and acoustic characteristics of the ported shroud compressor operating near surge. Although authors [16] attempted to predict the value of surge frequency, relatively lower surge frequencies are seen in literature as the upstream and downstream duct volumes were not considered by the authors in the numerical model. Rotating stall is also identified and is shown to propagate circumferentially at 0.5 RO. Dynamic mode decomposition of the transient numerical data $[15,16]$ pointed the interaction of flow exiting PS cavity as the possible cause of the rotating stall.

The analysis of the flow behaviour in the compressor operating near surge is critical to understand the corresponding acoustic characteristics and noise sources. The scope of experimental investigations using measurement probes or flow visualisation techniques such as laser Doppler velocimetry and particle image velocimetry are limited by the confined space and accessibility, particularly in automotive turbocharger compressors. The inception of the stall and development of surge in the ported shroud compressors is yet to be satisfactorily understood. Furthermore, the lack of quantification on the impact that ported shroud casing treatment has on the compressor acoustics dictates the need of this work. The current investigation rounds on understanding the flow behaviour and acoustic characteristics of the ported shroud compressor operating near surge (soft surge).

\section{Methodology}

Internal (in-duct) pressure approach $[6,14]$ is employed in this work to investigate the flow characteristics and compute the acoustic spectra of the compressor operating near surge. The methods like external radiated noise $[4,5]$ and orifice noise [10] are not considered due to additional computational overhead required for solving the noise propagation and noise radiation 
Table 1: Details of the modelled compressor

\begin{tabular}{lr} 
TC design intent & Commercial vehicles \\
Power & $400-600 \mathrm{~kW}$ \\
Number of blades & 7 main +7 splitter \\
Inducer to exducer ratio & 0.67 \\
Diffuser area ratio & 0.62 \\
\hline
\end{tabular}

from the compressor ducts. Moreover, the acoustic characteristics computed by internal pressure approach are well correlated with orifice noise as demonstrated by Torregrosa et al. [18].

The internal pressure fluctuations are numerically determined by solving unsteady Navier-Stokes equations for each control volume of the computational domain in their conservation form using a commercial CFD coupled solver, ANSYS CFX [19]. The computed pressure fluctuations are then processed using suitable approaches to obtain the noise levels at specific locations including the ducts of the compressor. Statistical Reynolds averaged and scale-resolving methods of turbulence modelling like Unsteady Reynolds Averaged Navier-Stokes (U-RANS) simulation, Large Eddy Simulation (LES) and Hybrid RANS-LES methods are explored in this work to model the near surge operation of the compressor.

Statistical methods rely on the Reynolds averaged form of Navier-Stokes equations to model all the scales of turbulence field. The flow solution is assumed to be composed of a mean and a fluctuating part, and the procedure solves directly for the mean flow solution. The time dependent mean component of the flow solution for URANS is achieved through the idea of separation of scales and is calculated by ensemble averaging. The Reynolds stress tensor introduced by the Reynolds averaging formulation is modelled using a two-equation Shear Stress Transport $(k-\omega$ SST) closure model.

Scale resolving methods focus on resolving all or a part of turbulent spectrum in at least a portion of grid. The first such method explored in this work is LES and the rationale of LES is to filter the time dependent Navier-Stokes equation to a certain scale in the physical space. The turbulent eddies or vortices
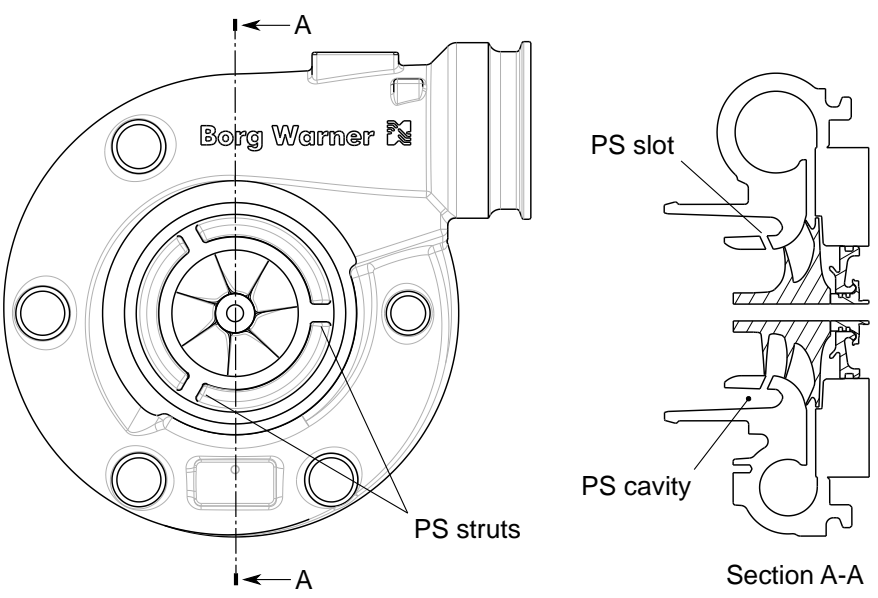

Section A-A

Figure 1: Mechanical drawing of the selected ported shroud compressor, showing the location of the supporting struts, the slot and the cavity geometry.

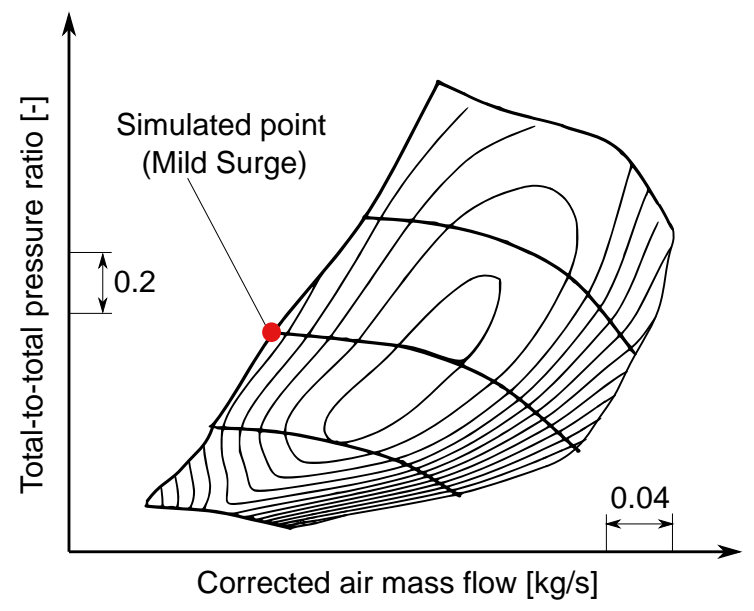

Figure 2: Experimental compressor map for the selected turbocharger, highlighting the near or mild surge operating point selected for the study.

smaller than the filter scale are modelled by appropriate means (sub-grid scale) while the larger eddies and coherent structures are resolved. The use of LES to model near-wall turbulence is particularly difficult because of the small turbulent length scales values and reverse direction of energy cascade. For the flows with high Reynolds number, the computational difficulty to resolve near wall regions is further increased due to the decrease in the size of viscous sublayer relative to the boundary layer with increase in Reynolds number. As turbomachinery problems are mostly wall-bounded flows at high Reynolds number, the LES approach would necessitate high spatial resolution. Although one could use wall-modelled LES approaches as an alternative but in this work Wall-Adopted Local Eddy-viscosity (WALE) [20] model formulation in CFX [19] is used with an intent to resolve the flow till viscous sublayer assuming the wall resolution is enough. The WALE-LES uses algebraic local eddy viscosity based sub grid model to dissipate eddies in viscous sublayer and near wall regions. This model improves upon the problem of the non-zero eddy viscosity in the laminar shear flow region seen in the Smagorinsky model.

Computationally efficient scale resolving alternatives to the LES are Hybrid RANS-LES methods in which the use of LES formulation is limited to large separation zones and for free shear flow. The mildly separated and attached boundary layers where LES requires excessive spatial and temporal resolution are modelled using RANS formulation. One of the relatively simple and widely used Hybrid method is Detached Eddy Simulation (DES). The switch between RANS and LES in the based on the local grid size. Therefore, the conventional DES formulation are highly grid dependent and suffers from the deficiencies like log-layer mismatch and grid-induced separation. These limitations are alleviated by incorporating shielding and blending functions in the conventional formulation of the DES. The conventional formulation of DES is extended to Delayed DES (DDES) in order to 'shield' the boundary layer from the DES limiter. The DES-SST [21] formulation in the CFX includes the shielding properties of DDES by formulating the blending functions of the SST model as the zonal DES limiter. 


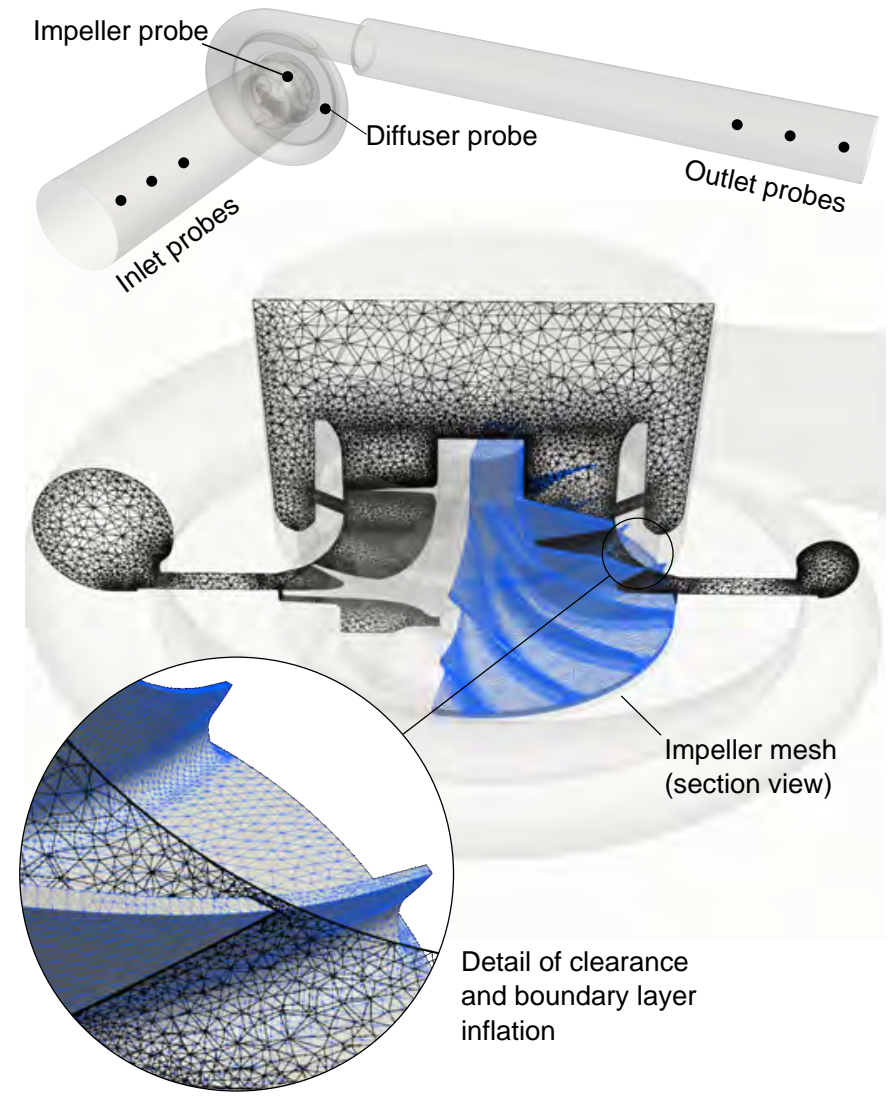

Figure 3: View of the computational domain focusing on the compressor, highlighting a slice of the fluid mesh and including the rotor surface mesh. A detail view shows the clearance between the blades and the shroud and the boundary layer inflation. Positions of the various axial pressure probes used to compute the acoustic spectra are also indicated.

The scale resolving Stress Blended Eddy Simulation (SBES) [22] model is also an improvement over conventional DES, specifically in the shielding of the boundary layer and transition issues in separating shear layers. The Reynolds stress tensor in the RANS region of the Hybrid formulation is again modelled using a two-equation Shear Stress Transport ( $k-\omega$ SST) closure model. Both DES-SST formulation and SBES model are explored to model the compressor flow.

The turbulence is initalised as isotropic with the curvature correction in the regions where Reynolds averaged method is used. The air used as working fluid in the compressor is assumed to be a perfect gas with the ideal gas law calculating the local density variation and Sutherland's law approximating the dynamic viscosity. The impeller motion is modelled using the Rigid Body Motion (RBM) approach, also known as sliding mesh in which the mesh actually rotates every time-step at the transient rotor-stator interface [19]. Boundary condition invloving the combination of total pressure at the inlet and static pressure at the outlet boundary is commonly used to model the flow in a compressor. However, it has been shown to be sensitive to an initial guess [19]. Therefore steady boundary condition of the total pressure at the inlet and mass flow rate at the outlet, is prescribed in this work to model the compressor operation at near surge condition. The values of mass flow rate and pressure used as boundary conditions are determined experimentally under steady flow operation. Besides, smooth walls with adiabatic and no-slip boundary are considered.

The computed in-duct pressure fluctuations are recorded at the multiple locations in the compressor using various virtual axial probes. The recoded pressure traces are then treated to obtain the spectral characteristics. Inlet and outlet duct spectra are computed from the decomposed pressure waves obtained using the Linearly-Constrained Minimum Variance (LCMV) beamforming [14] and Method of Characteristics (MoC) [23]. The spectra of the diffuser and near impeller locations are directly calculated from the pressure fluctuations recorded by the probes. The pressure wave decomposition at the duct is used to alleviate the issues such as the reflection from the duct ends and the standing waves. Beamforming uses two linear arrays of three axial pressure probes each at the inlet and the outlet duct to decompose the pressure wave into a forward travelling wave $p_{\text {forw }}(t)$ and backward travelling wave $p_{\text {back }}(t)$. The beamforming method assumes the plane wave propagation which is limited till the onset of first asymmetric mode $f_{c, a}$ defined in Equation 1. Another limiting range for the beamforming is given by the Nyquist-type frequency criterion $f_{c}$, which is based on the distance between the pressure sensors to avoid the aliasing problems. The experimental and numerical results for the inlet and outlet ducts are decomposed using beamforming.

$$
\begin{aligned}
f_{c, a} & =1.84 \frac{a}{\pi D} \sqrt{1-M^{2}} \\
f_{c} & <\frac{f_{i}}{2}=\frac{a}{2 L}
\end{aligned}
$$

The information of the flow field can also be used to reconstruct the pressure components by using the MoC. The pressure components are calculated in accordance to Equation 3 and Equation 4

$$
\begin{aligned}
& p_{\text {forw }}(t)=p_{\text {ref }}\left[\frac{1}{2}\left(1+\left(\frac{p}{p_{\text {ref }}}\right)^{\frac{\gamma-1}{2 \gamma}}\left(1+\left(\frac{\gamma-1}{2}\right) \frac{u}{a}\right)\right)\right]^{\frac{2 \gamma}{\gamma-1}} \\
& p_{\text {back }}(t)=p_{\text {ref }}\left[\frac{1}{2}\left(1+\left(\frac{p}{p_{\text {ref }}}\right)^{\frac{\gamma-1}{2 \gamma}}\left(1-\left(\frac{\gamma-1}{2}\right) \frac{u}{a}\right)\right)\right]^{\frac{2 \gamma}{\gamma-1}}
\end{aligned}
$$

In contrast to beamforming, MoC is not limited by any such inherent constraints and therefore, is used to compute the duct spectra at higher frequencies. The respective pressure components viz. $p_{\text {back }}(t)$ for the inlet duct and $p_{\text {forw }}(t)$ for the outlet duct are further processed by Welch's overlapped segmented average to estimate the Power Spectral Density (PSD) for the respective ducts.

A deeper insight on the relationship between the flow field the acoustic emission of the compressor is sought with the aid of modal decomposition techniques, specifically through the socalled Proper Orthogonal Decomposition (POD) which separates time and spatial characteristics of the flow field evolution. 


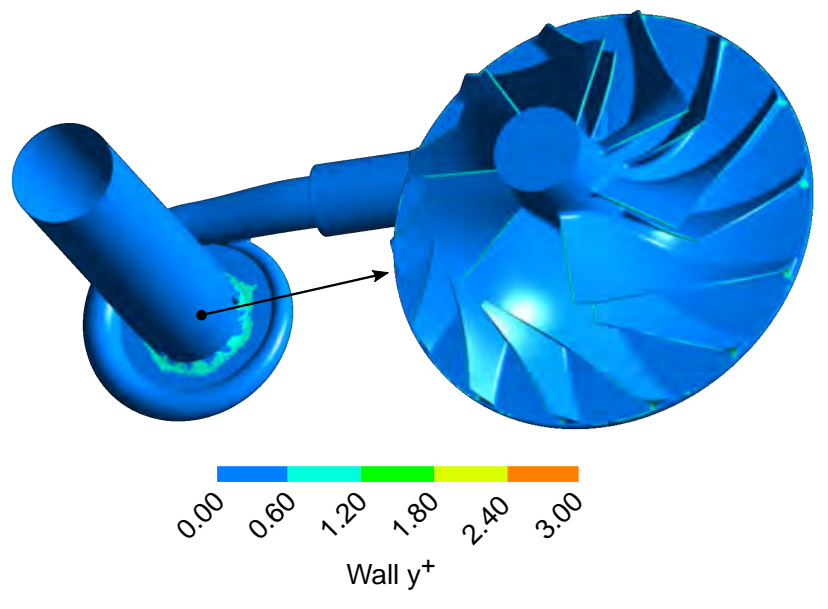

Figure 4: Contours of $y^{+}$across the domain walls. Except for some isolated cells with values up to 1.6 , only values less than 0.9 are seen in the shroud wall and blade leading/trailing edges.

\section{Geometry and computational model}

A commercially-available turbocharger compressor geometry with a PS recirculation treatment is the subject of this investigation. The front and section views of the compressor CAD geometry are shown in Figure 1. The PS cavity is structurally supported by three struts in a symmetric arrangement, and therefore, working fluid can enter or exit the PS slot via three channels. The impeller backside cavity (IBC) i.e. the gap between the impeller and the diffuser back-plate along with oil bearings has been modelled in this investigation. The specification of the compressor is provided in Table 1. The operation of the compressor at the near or soft surge condition of the isospeed line shown in the Fig. 2 is numerically investigated in this work.

The computational domain is spatially discretized by an unstructured polyhedral control volume created from the tetrahedral cells generated in the ICEM CFD [24] by the vertexcentered numerical approach in ANSYS CFX [19]. The grid is shown in the Figure 3. A polyhedral control volume is less diffusive and offers significant accuracy gains compared to an equivalent size unstructured grid of other cell types (i.e. tetrahedral) [25]. Although a structured grid would result in less numerical diffusion and commensurately higher accuracy at the cost of significantly higher user effort, it can also lead to cells with unavoidably large aspect ratios or heavy skewness in regions of high geometric complexity, resulting in unacceptable discretisation error. Furthermore, a polyhedral grid is expected to offer similar accuracy over equivalent hexahedral grid in the cases where secondary flows are relevant [26], and therefore, off-design operating conditions can be accurately modelled using a polyhedral grid.

The convective terms are discretised using a blend of second order accurate central difference scheme and first order upwind scheme to maintain the boundedness of the solution. An implicit, second-order accurate scheme implemented in ANSYS CFX [19] as second order backward Euler scheme is used to discretise the transient terms. It is worth noting that in this scheme the transient scheme for the turbulence equations is still first order, and a bounded second order scheme is used for the volume fraction equations. The hybrid model uses $1 \%$ turbulent intensity and a turbulent viscosity ratio of 10 at the inlet section. Five inner coefficient loops are used for each iteration to achieve
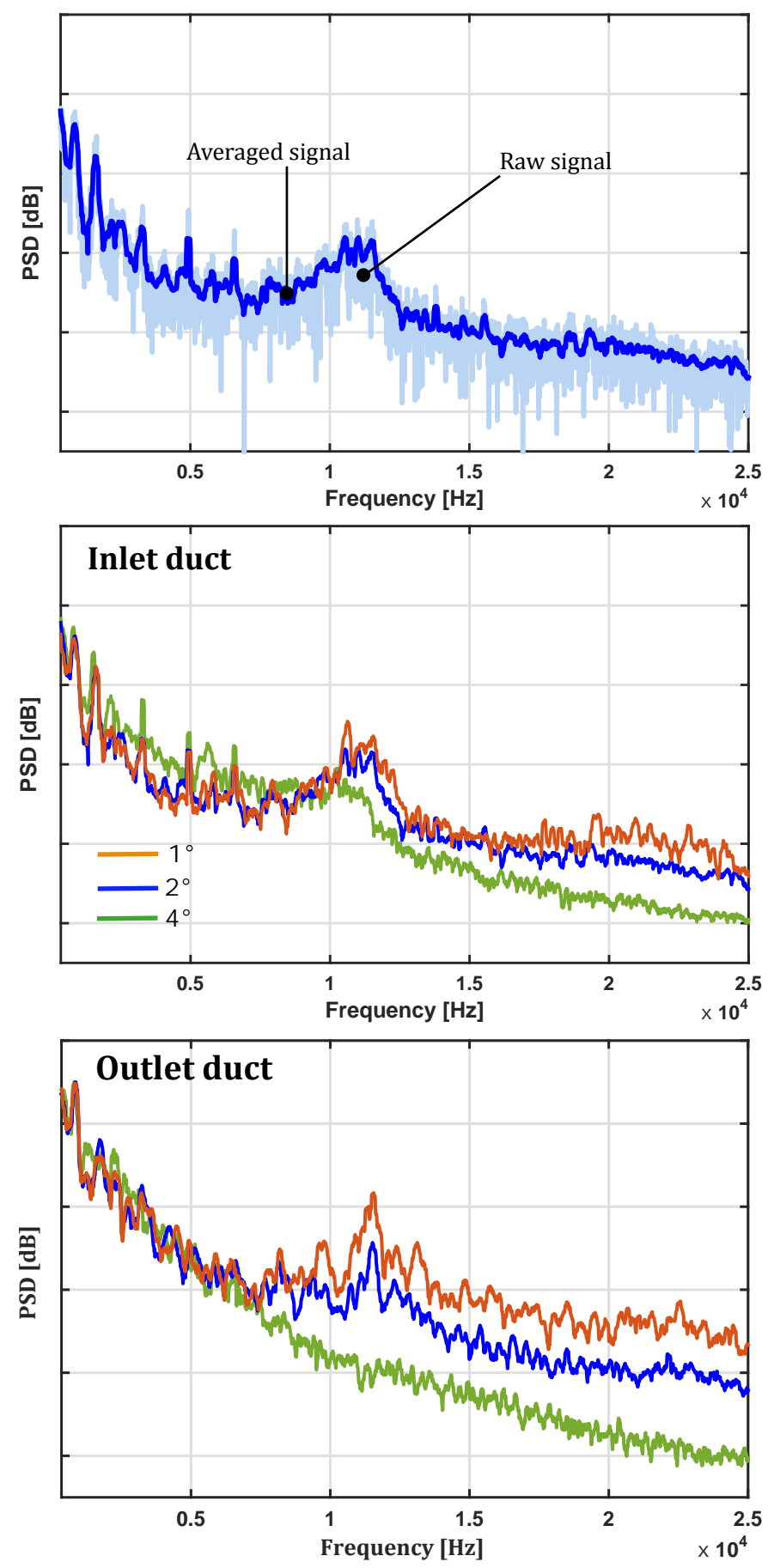

Figure 5: Impact of the timestep size $\left(4^{\circ}, 2^{\circ}\right.$ and $\left.1^{\circ}\right)$ on the acoustic predictions based on SBES model is illustrated. Inlet and outlet duct spectra is obtained by using the decomposing the pressure data recorded by the first sensor of each duct array using the MoC. Top plot shows an example of the averaged signal obtained from the central moving average procedure. Y-axis scale is kept at 20 $\mathrm{dB}$ per division. 
the convergence of residuals up to four orders $\left(10^{-4}\right)$. The inlet section of the compressor is extended by $3 D_{e}$ upstream and the outlet section of the compressor is extended by $6 D_{e}$ downstream to decrease the impact of the boundary conditions on the mean flow as well as to capture the flow instability that might propagate upstream of impeller.

The computational domain is assessed to yield a flow solution independent of grid density and time step by performing mesh independence and time step independence studies on successively refined spatial and temporal grids. The near-wall grids are investigated for their ability to resolve boundary layer flow, specifically the viscous sub-layer, by achieving a value of $y^{+}$closer to unity. Figure. 4 shows the global contours of $y^{+}$ values along with the $y^{+}$distribution at the impeller surface. It is worth mentioning that the grid independence study is performed using the URANS model and resulted in a grid with approximately 10 million cells. A grid with over double the number of elements ( 23 million) than the one finalised from grid independence study is then used to understand the impact of grid size on scale resolving models. The acoustic predictions from the higher density mesh shows an improved correlation with experimental results in terms of amplitude while the trend captured by both grids is similar. Therefore, the grid selected for this work consists of approximately 23 million cells and the boundary layer region is modelled using 12 prism layers growing exponentially with a growth ratio of 1.3.

Numerical configurations with the aforementioned turbulence modelling approaches and corresponding timestep sizes were investigated to model the operation of compressor. The detailed discussion on the impact of various turbulence models and the timestep sizes to model the near surge operation is beyond the scope of this article but the performance parameters (Eq. 6 and Eq. 7) predicted by various configurations are presented in Table 2. The impact of the timestep size on the prediction of the performance parameters is insignificant as seen from the results presented in Table 2. The duct spectra computed from the probes of the virtual duct array based on SBES model with timestep sizes corresponding to $4^{\circ}, 2^{\circ}$ and $1^{\circ}$ impeller rotation per iteration are presented in Figure 5 to briefly show the impact of the timestep size on the acoustics predictions.

It is important to point out that the local fluctuations in the estimated PSD spectra are smoothened by applying central moving average to the raw data all across this paper. Although, altering the parameters in the Welch's overlapped segmented average procedure can be used to smoothen or decrease the resolution of the spectra but that might compromise the low frequency region. For the dataset $\left\{g_{j}, h_{j}\right\}$ consisting of $v(j=1,2, \ldots, v)$ points where $g_{j}$ is an independent variable and $h_{j}$ is an observed value, the central moving average considering $\mathrm{u}$ adjacent data points is calculated as

$$
\left\{\mathbf{G}_{j+\frac{u}{2}}, \mathbf{H}_{j+\frac{u}{2}}\right\}=\left\{g_{j+\frac{u}{2}}, \frac{1}{j+u}\left(\sum_{i=1}^{j+u} h_{i}\right)\right\}
$$

An example of the application of moving average using 10 adjacent points on the raw PSD is shown in the Figure 5. The averaged signal demonstrates increased signal to noise ra-

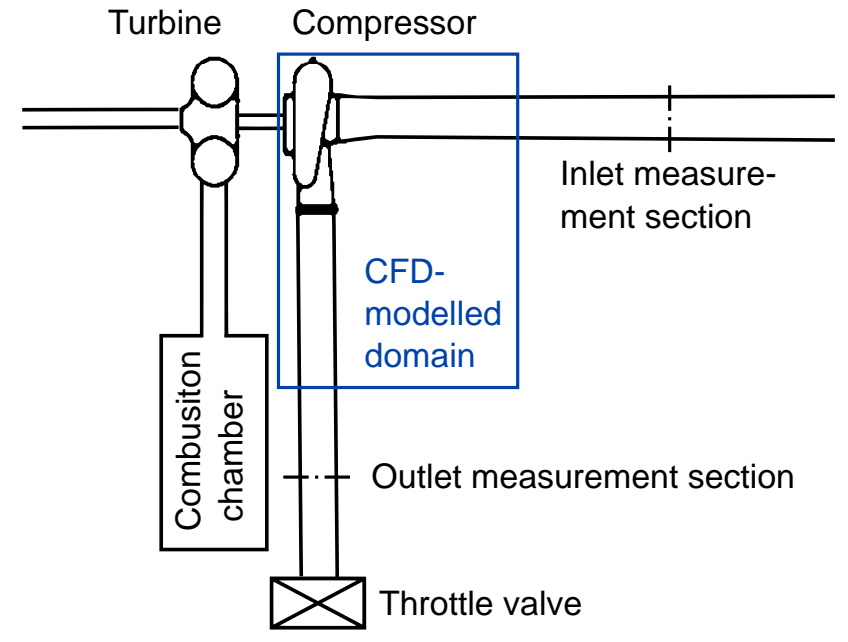

Figure 6: Sketch of the test rig used to experimentally characterize the compressor map, and to obtain boundary conditions and validation data.

tio without significantly distorting the raw signal and thereby, aiding in clearer visual inspection and comparison of the plots. The predicted spectra for the three models are similar up to a frequency of $8 \mathrm{kHz}$ while a significant deviation in the trends is observed at the higher frequencies for both inlet and outlet ducts. Broadband features like TCN present around $12-14 \mathrm{kHz}$ region is only identified in the outlet spectrum of $1^{\circ}$ timestep model. The difference in the overall levels can also be seen in the higher frequencies for different timesteps. An important inference that can be drawn is that the low - mid frequency phenomenon like 'whoosh' noise can be captured with relatively bigger timestep $\left(4^{\circ}\right)$ while high frequency features like the so called TCN can only be resolved by smaller timesteps $\left(1^{\circ}\right)$. The current work focuses on the noise content within the human hearing range. Therefore a time corresponding to $2^{\circ} \mathrm{im}-$ peller rotation is selected as the adequate time step size for the acoustic and flow analysis.

The macroscopic fluid structures which carry the most energy are the focus of the modal decomposition exercise in this work. These large scale structures are observed to be well resolved in the grid consisting of 10 million cells using SBES configuration with the timestep corresponding to $4^{\circ}$ impeller rotation. Therefore, on the basis of the computational and storage memory overheads, LES model on the grid consisting of 23 million cells and $2^{\circ}$ timestep is used for computing flow and acoustic characteristics while the SBES model on the 10 million gird with $4^{\circ}$ timestep is used for the modal decomposition campaign.

\section{Validation of the numerical model}

The computational model is validated in a two-step approach by first directly comparing the predicted performance parameters with the corresponding experimentally measured values. In the second step, acoustic results are validated by comparing the numerically computed spectrum with the experimental results. The above mentioned steps are elucidated in the subsections below. 
Table 2: Performance variables $\Pi_{t-t}$ and $\eta_{s}$ for the near surge operation predicted by various numerical configurations are compared with the experimental results. The numerical configurations used in this paper are also highlighted.

\begin{tabular}{llllll}
\hline & $\Delta t\left[^{\circ}\right]$ & $\Pi_{t-t}[-]$ & $\eta_{s}[\%]$ & $\varepsilon_{\pi}[\%]$ & $\varepsilon_{\eta}[\%]$ \\
\hline \multirow{2}{*}{ Experiment } & - & 2.47 & 66.7 & - & - \\
\hline \multirow{2}{*}{ URANS } & $4^{\circ}$ & 2.44 & 66.0 & 1.2 & 1.0 \\
& $2^{\circ}$ & 2.44 & 65.9 & 1.2 & 1.2 \\
\hline \multirow{2}{*}{ DES } & $4^{\circ}$ & 2.46 & 66.1 & 0.4 & 0.9 \\
& $2^{\circ}$ & 2.44 & 66.0 & 1.2 & 1.0 \\
\hline \multirow{2}{*}{ SBES } & $4^{\circ}$ & 2.47 & 66.4 & 0.1 & 0.5 \\
& $2^{\circ}$ & 2.45 & 66.2 & 0.8 & 0.7 \\
& $1^{\circ}$ & 2.44 & 65.9 & 1.2 & 1.2 \\
\hline \multirow{2}{*}{ LES } & $2^{\circ}$ & 2.48 & 66.5 & 0.2 & 0.4 \\
\hline
\end{tabular}

\subsection{Validation of the global performance predictions}

The first level of validation is achieved by comparing the performance parameters predicted by the computational model with gas stand measurement data. The global performance parameters of total-to-total pressure ratio $\Pi_{t-t}$ and isentropic efficiency $\eta_{s}$ are used to validate the numerical model.

$$
\begin{aligned}
\Pi_{t-t} & =\frac{p_{\text {out }, 0}}{p_{\text {in }, 0}} \\
\eta_{\mathrm{s}} & =\frac{\dot{W}_{\text {is }}}{\dot{W}}=\frac{T_{\text {in. } 0}}{T_{\text {out }, 0}-T_{\text {in }, 0}}\left(\Pi_{t-t}^{\frac{\gamma-1}{\gamma}}-1\right)
\end{aligned}
$$

Compressor measurements are performed at the gas stand testing facility in BorgWarner Turbo Systems, Bradford. The turbocharger test bench characterizes both the compressor and turbine as the turbocharger is tested as a whole machine. The test rig uses pressurized air as the working fluid, heated to $600^{\circ} \mathrm{C}$ in a combustion chamber. The mass flow rate of the compressor is altered by controlling a throttle valve located at the end of the compressor outlet duct. Mass flow rate, ambient temperature and ambient pressure are measured at compressor inlet while static/total pressure and temperature are measured at the compressor inlet and outlet sections. Turbo shaft speed is measured using the machine's own speed sensor. Surge (both mild and deep) is detected using an automated method based on the compressor inlet pressure fluctuations. Data is generally sampled at a frequency of $100 \mathrm{~Hz}$ except for inlet pressure that is measured at $1 \mathrm{kHz}$ to detect surge. Absolute error in temperature measurement is in the range of $\pm 1{ }^{\circ} \mathrm{C}$. The repeatability of pressure ratio values, rotational speed and mass flow rate measurements are within $\pm 1 \%$. The influence of the instrumentation on the compressor flow is minimized by placing the probes at suitable distance upstream and downstream of compressor i.e. 4-8 times the duct diameter. The simplified schematic of the test rig is shown in Figure 6.

The time averaged total-to-total pressure ratio $\Pi_{t-t}$ and isentropic efficiency $\eta_{s}$ values from numerical model and gas stand measurement are compared in the Table 2 along with relative error $\varepsilon$

$$
\varepsilon=\frac{\left|\phi_{\text {num }}-\phi_{\exp }\right|}{\phi_{\exp }} 100
$$
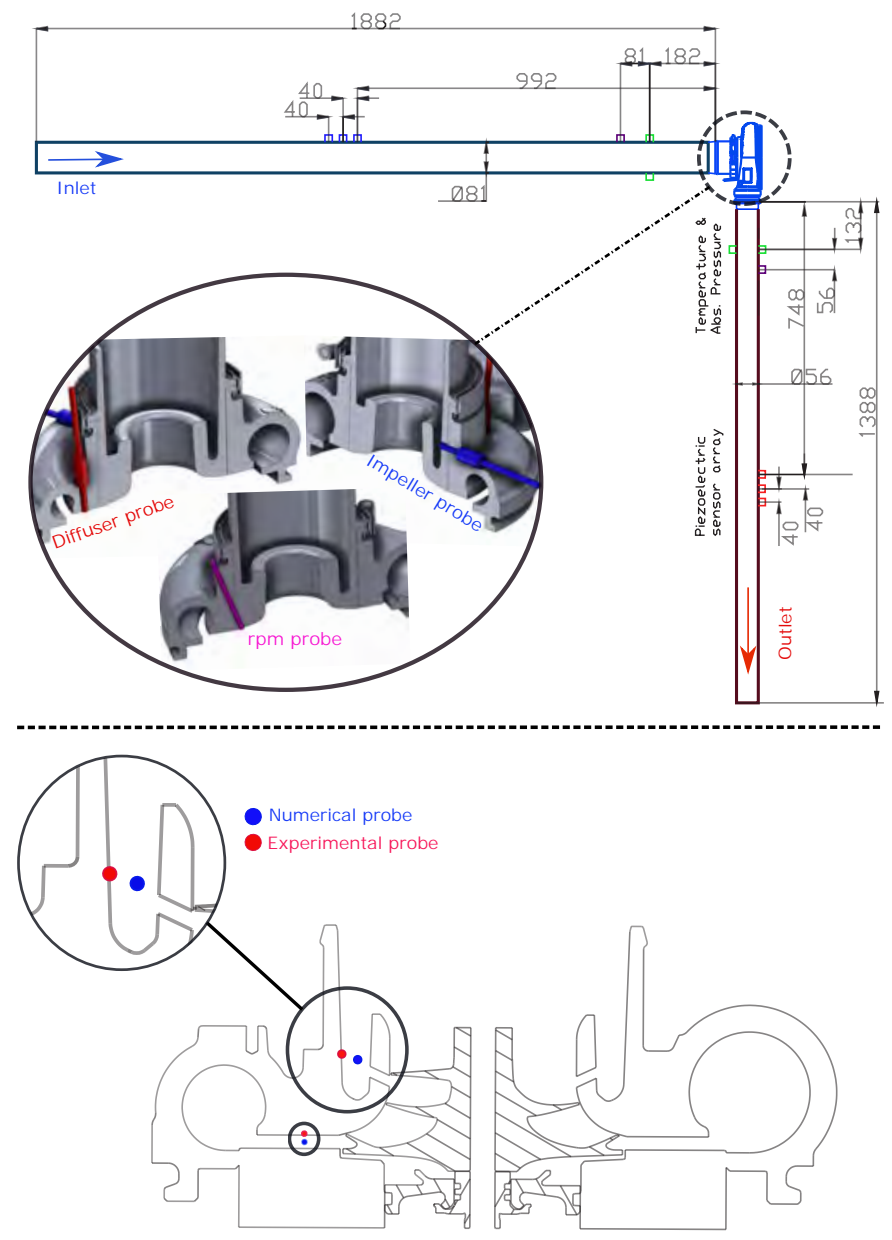

Figure 7: Schematic of the test rig used to determine the acoustic characteristics of the compressor is shown (dimensions in $\mathrm{mm}$ ). The location of various pressure and temperature sensors on the rig is marked. Impeller and diffuser sensor instrumented on the compressor are shown in the detailed view. The bottom figure highlights the difference in the location of experimental (wall flushed) and numerical (mid flow) probes.

It is worth pointing out that the numerical simulations are carried out for approximately 165 impeller revolutions of which about 35 compressor revolutions corresponds to the intial transient state. Therefore, the numerical results are averaged over around 130 compressor revolutions which corresponds to about 0.08 s while experimental values are averaged over $1 \mathrm{~s}$ period. The numerical performance parameters are slightly under-predicted, which seems counter intuitive as the walls are modelled smooth and adiabatic. Literature [27] shows that modelling the IBC results in the lower values of the performance variables computed in the numerical model. Nevertheless, the computed results are in close agreement with the gas stand measurements.

\subsection{Validation of the acoustics predictions}

The acoustic spectra computed from the pressure signal of the probes located near impeller inlet and downstream of the impeller are compared to the experimentally measured spectrum at the similar location. The experimental measurements are carried out by installing the turbocharger on the test rig facility in CMT-Motores Térmicos, wherein exhaust from an 

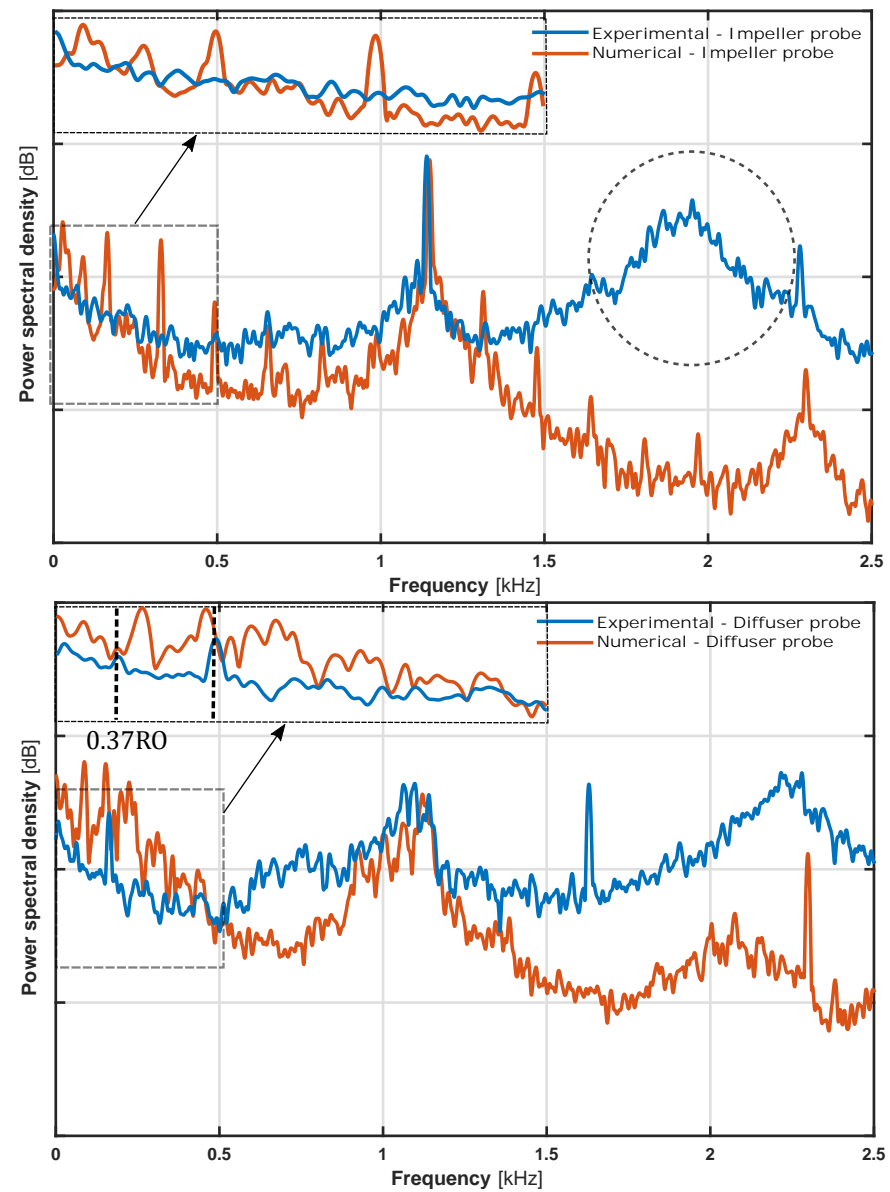

Figure 8: Comparison of the PSD obtained from numerical and experimental exercises for the near impeller (top) and the diffuser (bottom) probe. A close-up of the spectra till $5 \mathrm{kHz}$ is also presented for both probes. Y-axis scale is kept at $20 \mathrm{~dB}$ per division.

engine is used to run the turbine of the turbocharger. The measurements are made with idealised long straight pipes at the inlet and the outlet openings of the turbocharger. The position of piezoelectric pressure sensors used to measure the pressure fluctuations near impeller and in diffuser are shown in the Fig. 7 along with the schematics of the test rig. The pressure signal is sampled at $200 \mathrm{kHz}$ for $1 \mathrm{~s}$ at the appropriate operating condition using a Yokogawa digital oscilloscope. The equivalent pressure signal from the numerical model are recorded for $0.1 \mathrm{~s}$ ( $\sim 165$ compressor revolutions) including $0.02 \mathrm{~s}$ ( $\sim 35 \mathrm{com}-$ pressor revolutions) of initial transient. The pressure signals are then processed by Welch's overlapped segmented average to compute the estimated PSD at the corresponding positions. Hamming windowing is used at blocks with $50 \%$ overlap and a frequency resolution of $50 \mathrm{~Hz}$ is achieved.

The correlation of the sound spectra predicted by the numerical configurations with the measured spectrum are presented in Figure 8. Blade pass frequency (BPF) and the harmonics of BPF are correctly identified in the predicted spectra of both near impeller and diffuser probes. Rotor shaft speed harmonics ('buzz-saw' tones) are significantly accentuated in the numerical spectra as compared to the experimental spectra. The ex- perimental spectrum of the near impeller probe is dominated by the BPF with relatively straight overall levels with the broadband in the region of $17-22 \mathrm{kHz}$ which is not captured in the numerical spectrum. Similarly, overall level seen in the experimental spectrum of diffuser probe is also not captured by the numerical result although the trend shows a reasonable agreement. The limitations of the numerical results includes the deviation in the overall levels and the inability to capture above mentioned broadband noise.

The deviation of the numerical spectrum from the measurement can partly by attributed to the location of the pressure sensor. The probe used in experiments are wall flushed as shown in Fig. 7 while the pressure in numerical model is monitored in the middle of the cavity. The constraint of mid-flow probes in the numerical model is based on the necessity of particularly high spatial resolution in LES approach to accurately model the wall-bounded flows at high Reynolds number. The boundary layer effects captured in the experimental measurements are ignored in the numerical model. It may be the case that more advanced turbulence models are required to further increase the correlation, and it may also be the case that the origin of this white noise seen in experimental results is related to fluid-solid interactions (blade flutter, axis torsion/deflection, etc.) that are not modelled. Nevertheless, the model credibly predicts the trend and reasonably replicates the features seen in the experimentally measured acoustic characteristics.

\section{Results and discussion}

\subsection{Compressor spectral signature}

Power Spectral Density (PSD) of the probe near impeller for the modelled turbocharger compressor is shown in Figure 9. As mentioend before, the tonal features like Blade Pass Frequency (BPF), harmonics of BPF and the harmonics of rotor shaft speed ('buzz-saw' tones) are observed in the spectrum. The strength of the tonal features in the near impeller spectrum

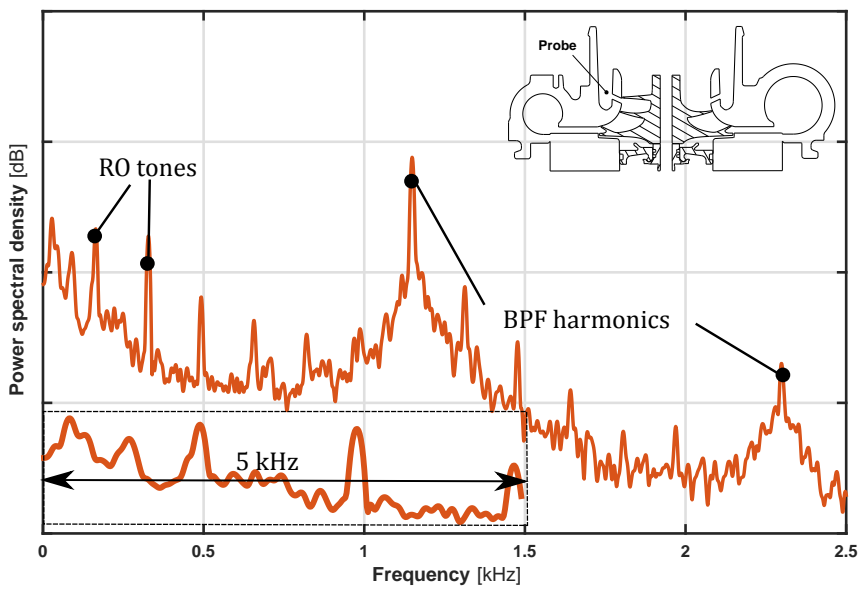

Figure 9: Spectrum at the near impeller location predicted by the numerical model. A close-up of the spectrum till $5 \mathrm{kHz}$ is also shown. Y-axis scale is kept at $20 \mathrm{~dB}$ per division. 
are relatively weaker for near surge operation as compared to the design operation [28].

It is interesting to note that the faint 'mid-tones' [28] that are present between the 'buzz-saw' tones in the near impeller spectrum for the design operation are not existent in the spectrum of the compressor operating near surge. The broadband in the frequency range corresponding to characteristic 'whoosh' noise region is not seen in the spectrum of the near impeller probe as observed in the close-up sections presented in Figure 8 and Figure 9. Nonetheless, characteristic 'whoosh' noise may not outstand but compared to other points far from surge, an increase in the noise levels at lower frequency range are observed. A tonal feature corresponding to $0.37 \mathrm{RO}$ is observed in the numerical and measured spectra the diffuser probe. Similar feature can be also be identified in the numerical spectrum of the inducer probe but its presence in the measured inducer spectrum is not significantly substantiated. This peak corresponds to the rotating stall and is expected to be caused by the propagation of the stall cells around the impeller with similar speed as shown in the later part of this article.

Power Spectral Density (PSD) at the pressure probes located $90^{\circ}$ apart circumferentially in the middle of the diffuser along with a probe near the volute tongue is shown in Figure 10. A weak presence of BPF, its first harmonic and RO tones are observed in the spectra of all the probes located in the diffuser. The spectra of these probes including the probe located near the volute tongue are quite similar. It is interesting to the point that the amplitude of pressure fluctuations captured by the 3rd probe (opposite to the volute tongue) is lower than rest of the diffuser points, but the sound level is similar to the remaining diffuser points.

\subsection{Inlet and outlet decomposed pressure spectra}

Pressure arrays positioned at the inlet and outlet duct of the compressor are used to store the temporal trace of the pressure for $0.08 \mathrm{~s}$ excluding the initial transients. The pressure fluctuations are decomposed and are processed by Welch's overlapped segmented average to compute the PSD at inlet and outlet ducts. The duct spectra are validated by comparing agaisnt the measured PSD in the low frequency region corresponding to the plane wave propagation of the sound. Experimentally measured pressure fluctuations are decomposed using LCMV beamforming based method to compute the duct spectra. Numerical results are computed from the pressure wave decomposed using beamforming as well as from the pressure wave reconstructed from the flow-field information using MoC) [23].

The plane wave propagation for the studied case is limited by the onset of first asymmetric mode. The limiting values for the inlet and outlet duct in the measured spectra are $2.5 \mathrm{kHz}$ and $4.25 \mathrm{kHz}$. The intent of decomposing the pressure wave is to enhance the content coming from compressor and to obtain the pressure fluctuations with lower reflected content from the boundary. Although non-reflecting boundary conditions and significantly longer ducts with successive grid coarsening can be used to address the reflection by numerical dispersion, these methods are not used in this study due to computational and numerical configuration reasons.

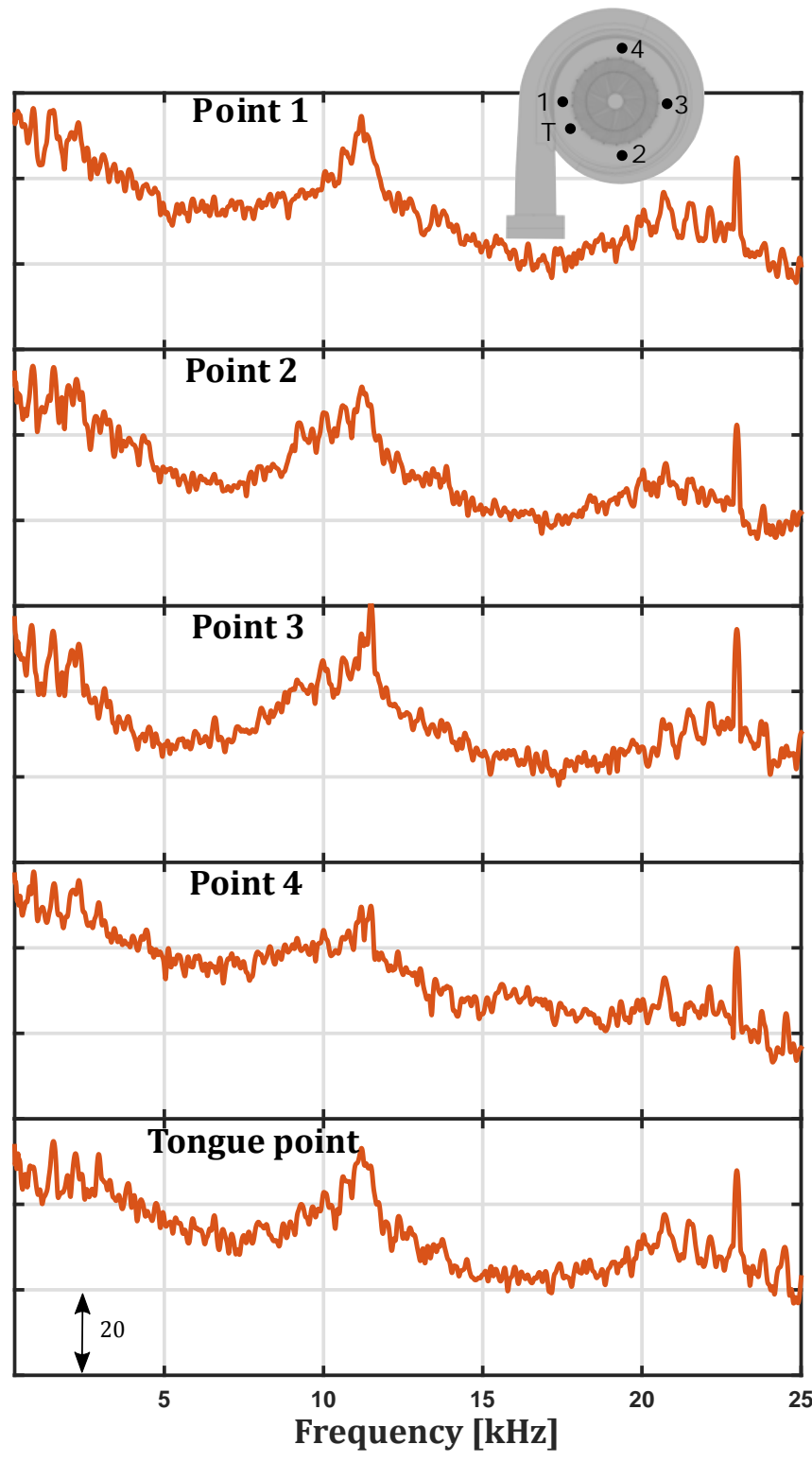

Figure 10: Result of predicted pressure spectral density (PSD) for different points in the diffuser, and near the volute tongue. Y-axis differences are arbitrary, although scale is kept at $20 \mathrm{~dB}$ per division.

The spectra of pressure components obtained using beamforming and $\mathrm{MoC}$ are compared with beamformed experimental spectra in the Figure 11. The spectra obtained from $\mathrm{MoC}$ and beamforming shows higher coherence for the inlet duct relative to the outlet duct. This makes sense as the flow properties are expected to be uniform at the inlet array while the presence of high swirl at the outlet of the compressor would introduce difference in the flow field recorded by the individual probes of the outlet array. Nonetheless, numerical results shows a reasonable correlation with the experimental results for the low frequency region.

The high frequency spectra of the inlet and outlet duct are obtained from the pressure traces reconstructed using MoC given the inherent limitations associated with the beamforming method does not apply. The experimentally measured duct spectra and 
the numerically predicted results are presented in the Figure 12. It is interesting to see the propagation of the first asymmetric mode in the experimental spectrum of the inlet duct. The presence of this mode has increased the level of the inlet duct PSD to that of the outlet duct unlike the numerical results, where a steady difference in the levels of inlet and outlet duct spectra can be seen. The asymmetric mode is not present in the numerical spectrum as the pressure wave is decomposed.

Blade pass frequency can be identified in the numerical spectra of both ducts although first harmonic of the BPF is not prominent in contrast to the measured spectra. Broadband noise features present after the BPF in the measured spectra are also not captured by the numerical model. Although the broadband in the outlet duct is captured by the numerical model with smaller timestep $\left(1^{\circ}\right)$ as seen in the Figure 5. The broadband feature observed in the outlet duct can be loosely connected to the so called TCN although TCN is usually identified before BPF in the literature [29] while the broadband observed in the measured spectrum of inlet duct is expected to be caused by the higher order duct modes and is thereby, no captured in the numerical spectrum. Furthermore, the decay of numerical spectra is stepper than what is seen in the experimentally measured results. The so called 'whoosh' noise is not clearly identified in the numerical or experimental spectra.
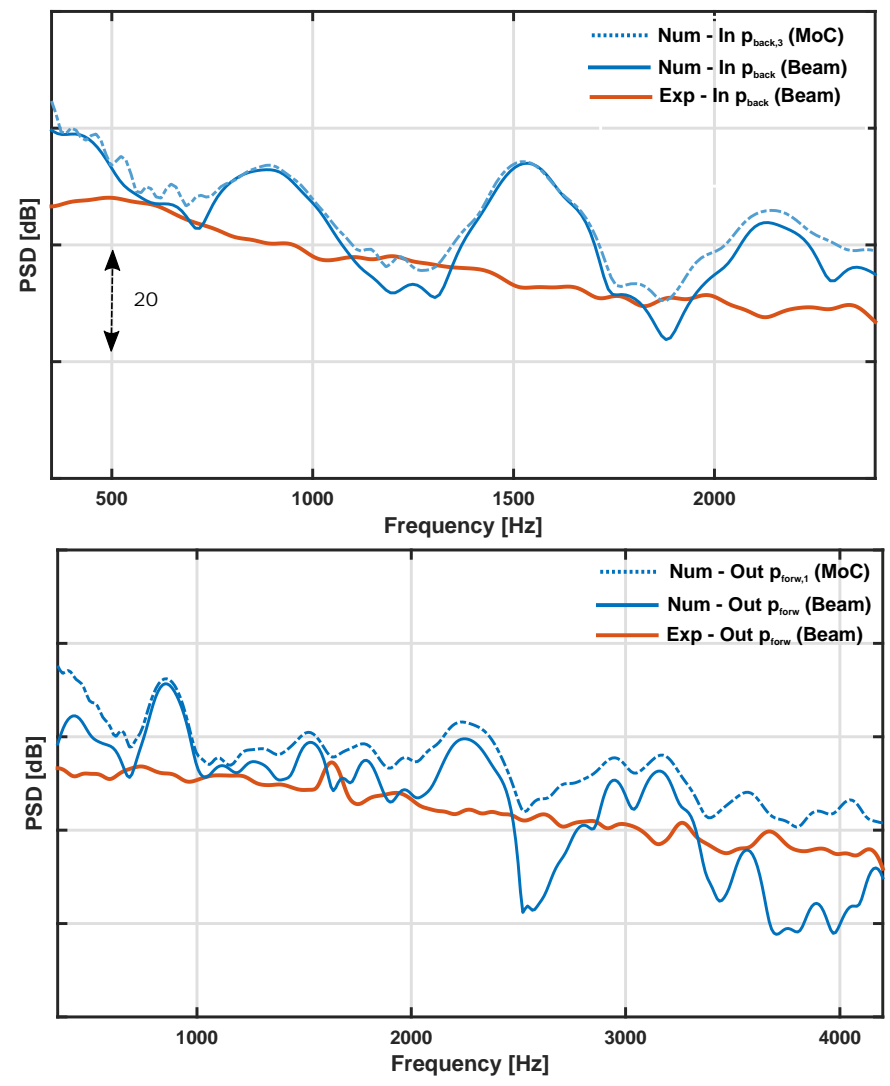

Figure 11: Spectra of the inlet- $p_{\text {back }}$ (top) and outlet- $p_{\text {forw }}$ (bottom) duct till plane wave limit are presented. Experimental spectra obtained from the pressure waves decomposed by LCMV beamforming are compared with the numerical results obtained from the decomposed pressure waves using beamforming and MoC.
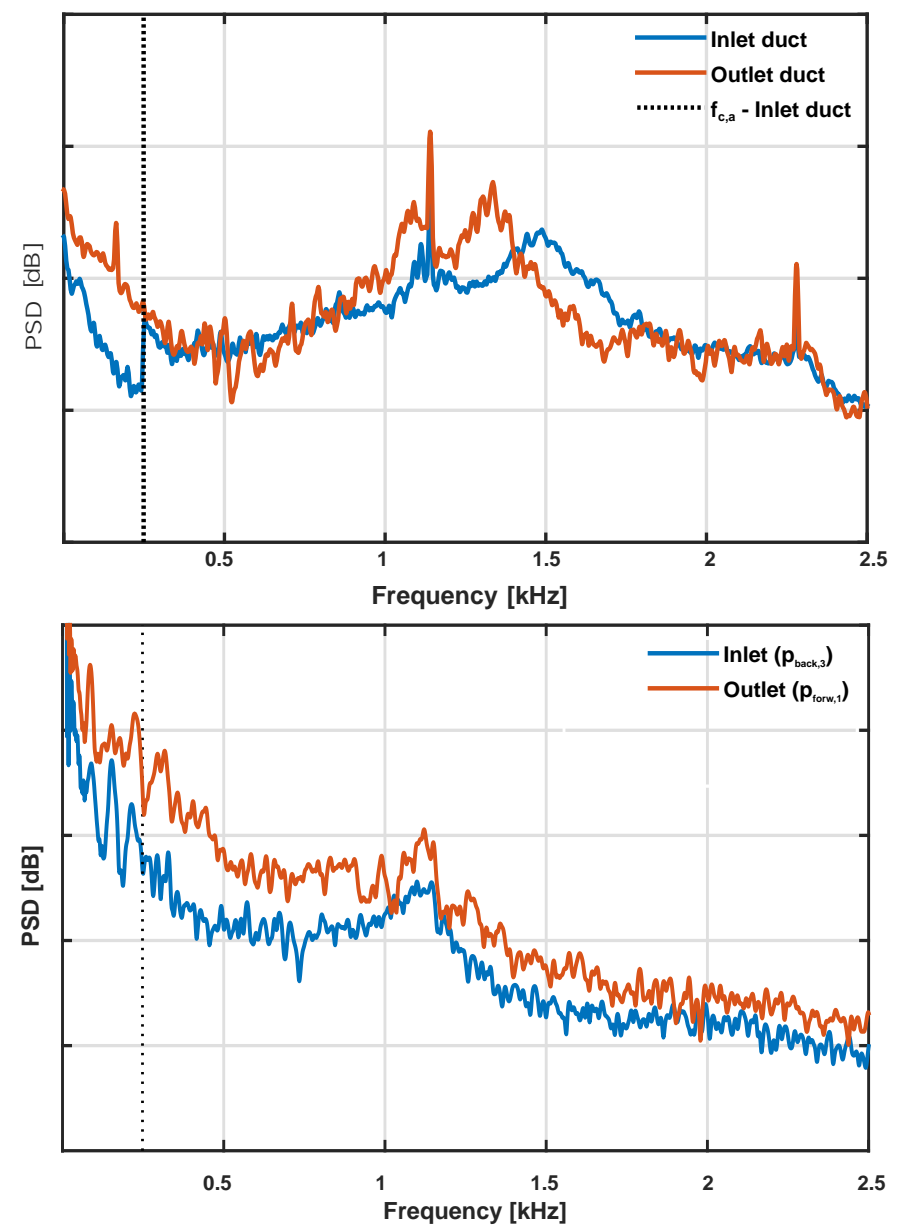

Figure 12: Inlet and the outlet duct spectra obtained from experimental (top) and numerical (bottom) probes. The experimental spectra is obtained from raw pressure signals while numerical spectra is obtained from pressure waves reconstructed using MoC. First asymmetric mode in the experimental spectrum of the inlet duct has increased the noise level unlike in numerical result. Y-axis scale is kept at $20 \mathrm{~dB}$ per division.

The agreement between the predicted and measured results for the compressor ducts is not good but the noise generation as seen from the spectra of the probe near impeller probe and in diffuser is reasonably captured. The deviation between the numerical and experimental results can be reduced by employing section probe in the numerical model instead of currently used point probes. The section probe would help in capturing the higher order effects introduced by swirl and related three dimensional flow features seen in the compressor. Also, the tuning of the duct dimensions used for the experimental measurements to minimise duct propagation effects can also help with decreasing the gap between experimental and numerical results.

\subsection{Flow field characteristics}

In this section, the flow field characteristics including the flow through the PS cavity for the compressor operating near surge condition are investigated. A significant fraction of inlet mass flow is seen to be recirculated through the PS cavity as previously predicted using steady approach [30]. The fluid 


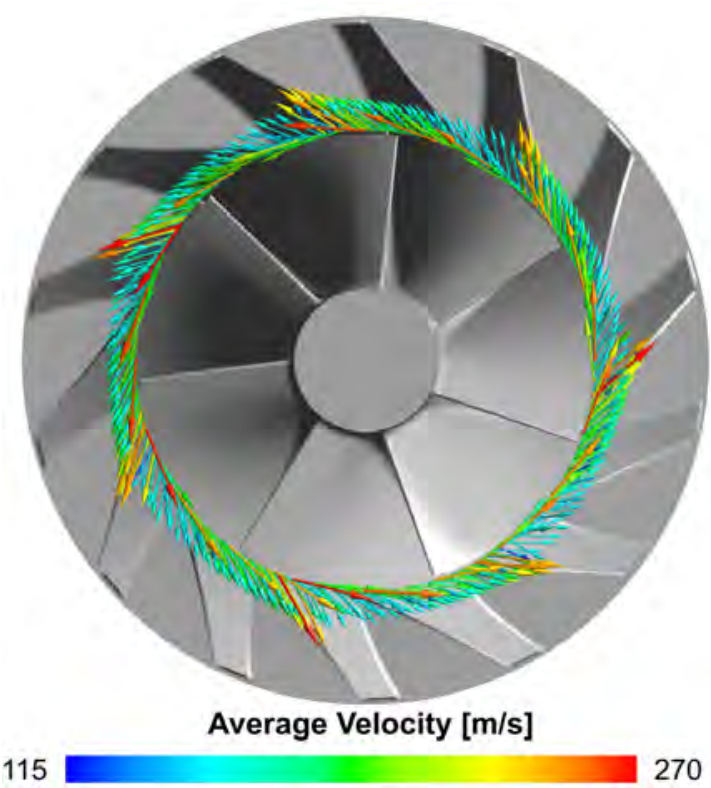

Figure 13: Average velocity vectors in the rotating frame at the PS slot location shows radially outward flow from blade passages into the PS cavity.

with high circumferential velocity is removed from the impeller passages and recirculated to the main flow via PS cavity. In contrast to the constant pull and push behaviour of the fluid along the impeller blades at the PS slot for design operation [28], a unidirectional radially outward flow is seen at the PS slot for the near surge operation as shown in Figure 13. The flow exits PS cavity in form of high speed jets corresponding to the structural struts. The vortices identified using $\lambda_{2}$ criterion presented in Fig. 14 clearly shows the turbulent features formed by the interaction of recirculated flow with the inlet flow. These turbulent structures are expected to be a significant source of the losses that are seen in the 'Inlet-PS' mixing region as second law analysis [30] points 'Inlet-PS' mixing region as the dominant sources of losses during near surge operation.

The flow structures arising due to the interaction in the mixing region decreases the effective area for the inlet flow and thereby, increasing the bulk axial velocity of the inlet flow as observed in the velocity streamlines presented in Figure 15. The increased temperature in the inlet region is also a direct consequence of the interaction between recirculated flow (reverse flow for non-ported shroud compressor) and the inlet flow as the recirculated flow is already worked upon by the impeller. The swirl added into the compressor inlet flow by the recirculated flow also significantly impacts the incidence at the leading edge of impeller blades.

The meridional velocity distribution in the impeller passages for $50 \%$ blade span at six time instances, each corresponding to $32^{\circ}$ impeller rotation is shown in the Figure 16. The propagation of multiple stall cells can be clearly seen in the plot by observing the flow behaviour in the specified blade passages $1,2,3$ and 4 . In the first time instance i.e. $\beta^{\circ}$, stall initiation and fully developed stall can be observed in the passage 1 and passage 2 respectively while passage 3 and passage 4 are

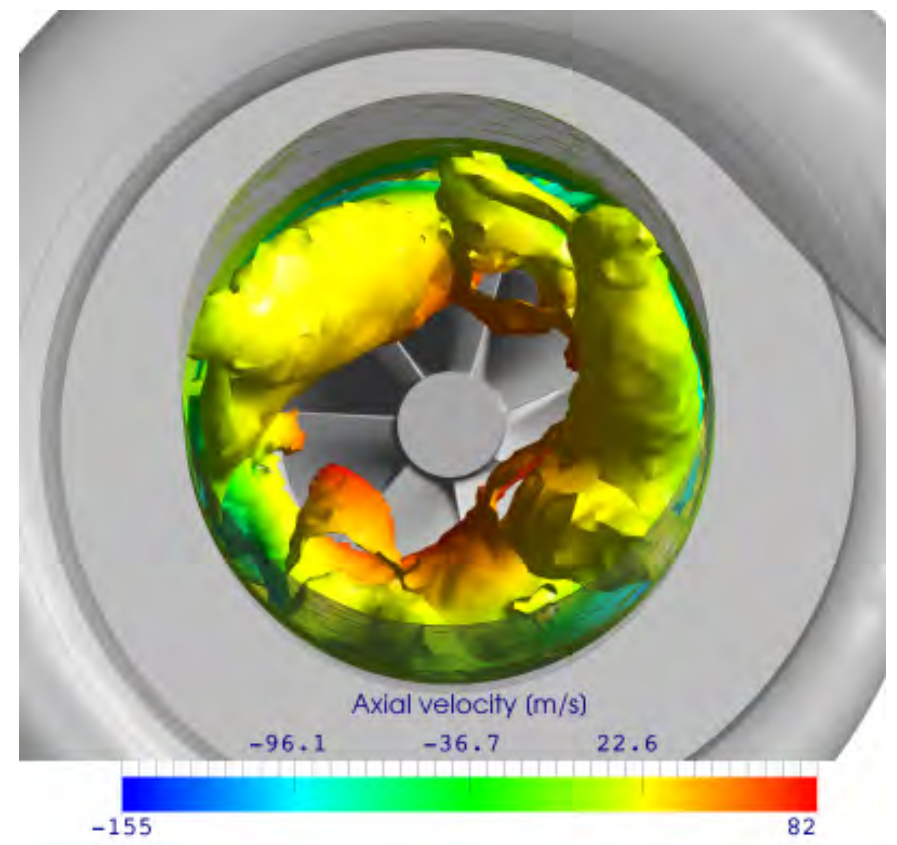

Figure 14: High speed jets corresponding to the struts are identified by $\lambda_{2}$ vortex criterion. The structures are coloured with average axial velocity.

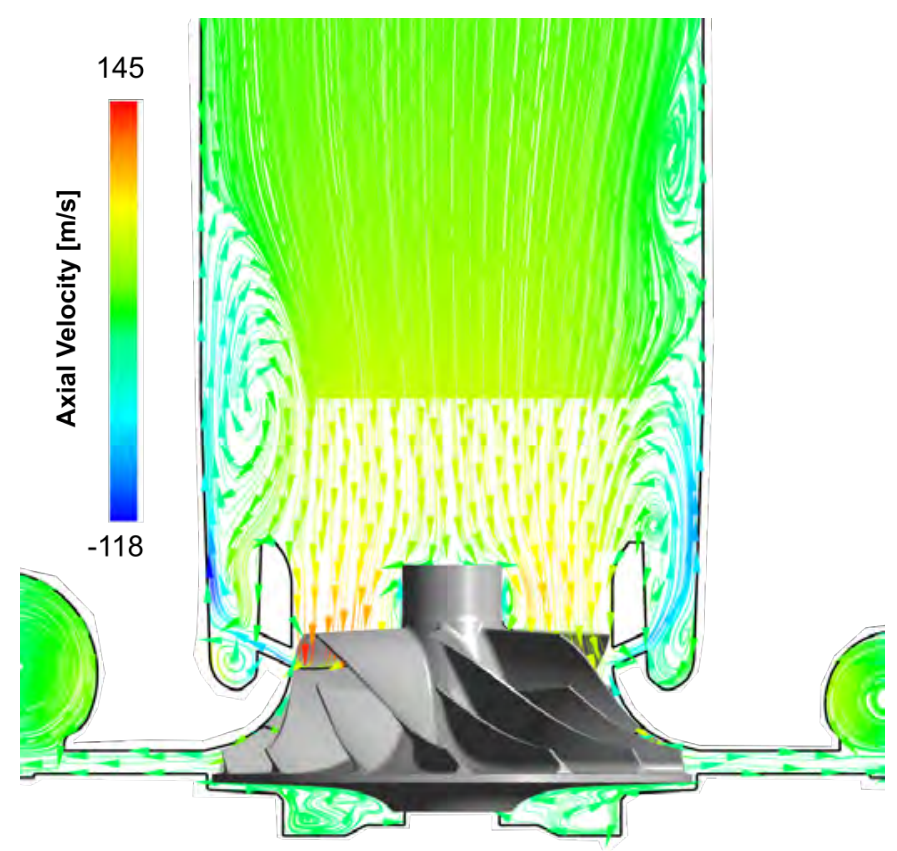

Figure 15: Flow field characteristics at the impeller axial plane. Surface streamlines coloured by average velocity magnitude demonstrate the turbulent features formed by the interaction of the recirculating and the inlet flow.

recovering from the stall. As the impeller blades rotates by $32^{\circ}$ i.e. $\beta+32^{\circ}$, development of stall can be seen in the passage 1 while the strength of the stall is decreased in the passage 2 , passage 3 and passage 4 . Similarly, by sixth time instance i.e. $\beta+160^{\circ}$, the blade passage 4 and passage 3 are free of stall while passage 2 and passage 1 are recovering from the stall. These stall cells are seen to propagate circumferentially around 

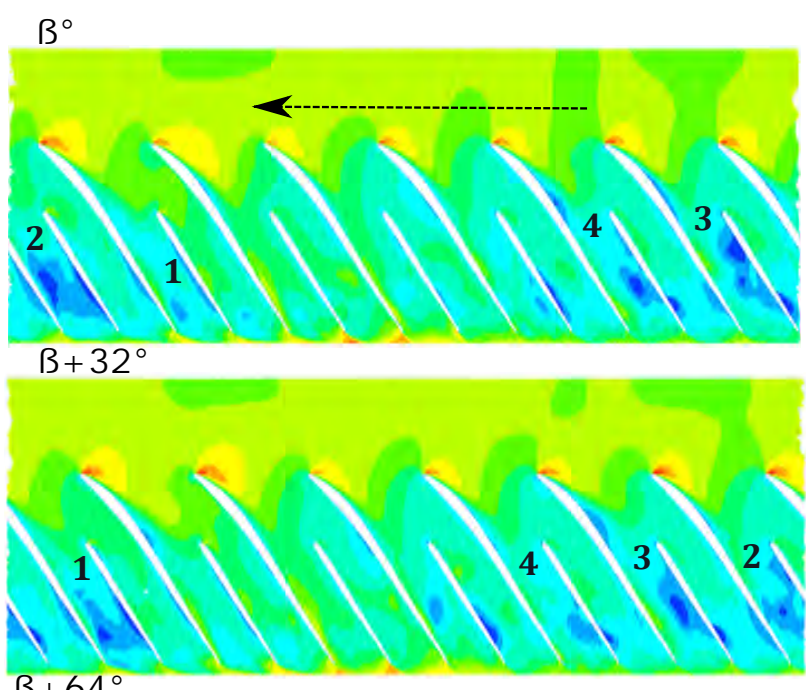

\section{$\beta+128^{\circ}$}
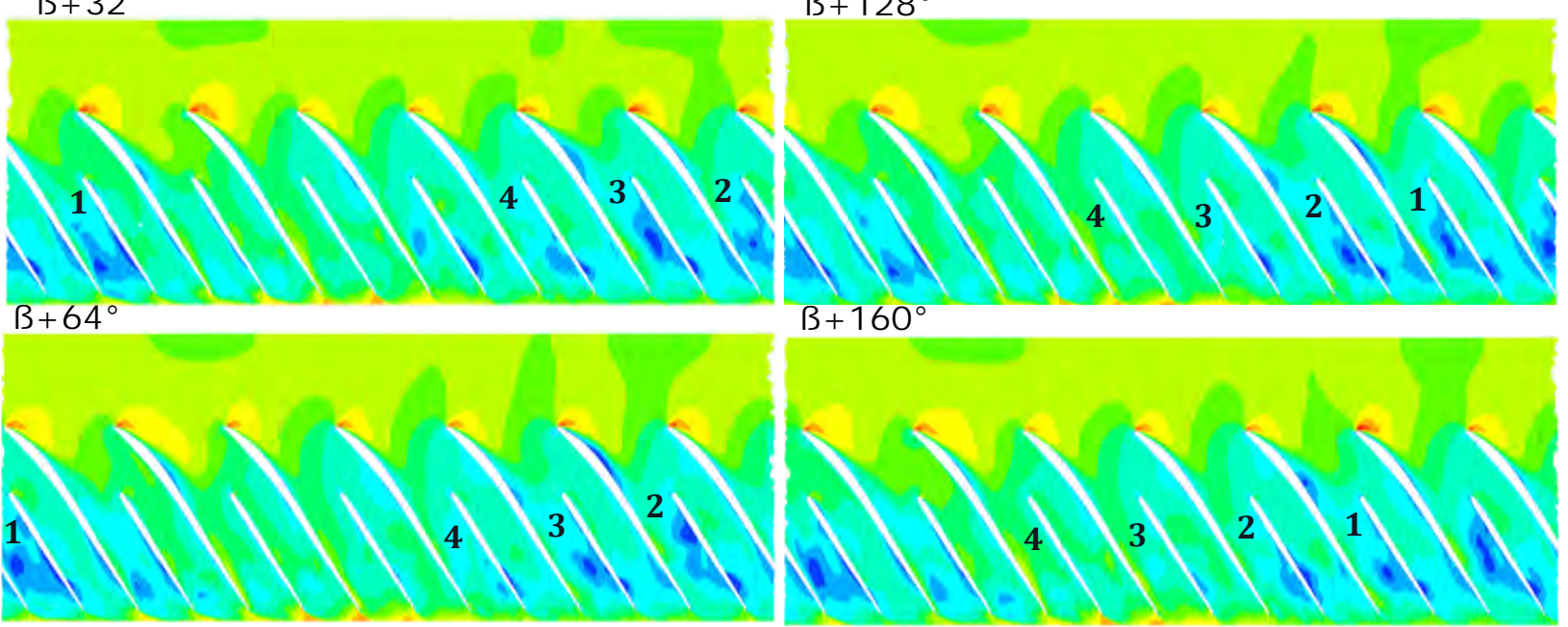

Velocity $[\mathrm{m} / \mathrm{s}]$

0

300

Figure 16: Stall cell propagation is demonstrated by colouring the $50 \%$ blade span surface with instantaneous meridional velocity for six subsequent impeller positions.

the impeller with a speed of approximately (0.32-0.4)RO which corresponds to the peak seen in the diffuser spectrum at $0.37 \mathrm{RO}$. Similar results identifying a tone at $0.5 \mathrm{RO}$ in the spectra of diffuser points for the near surge operation are reported in works of Jyothishkumar et al. [17] and Sundström et al. [16].

\subsection{Flow field modal decomposition}

In an effort to understand the relationship between flow structures and acoustic features, a data-based mathematical technique named as Proper Orthogonal Decomposition (POD) is employed to extract the spatial features called modes accompanied with the energy content levels [31]. Modes extracted by POD are based on optimising the mean square of the studied flow variable and in essence, is an algorithm to decompose data into a minimal number of basis functions to capture as much energy as possible.

The unsteady component of the flow field variable is represented in terms of a generalised Fourier series for some set of basis functions and an optimal set of those basis functions is computed in the POD. The unsteady component $\mathbf{x}(\xi, t)$ of a flow field variable $\mathbf{q}(\xi, t)$ with temporal mean $\overline{\mathbf{q}}(\xi)$ can be decomposed as

$$
\mathbf{x}(\xi, t)=\mathbf{q}(\xi, t)-\overline{\mathbf{q}}(\xi)=\sum_{j} \mathbf{a}_{j} \phi_{j}(\xi, t)
$$

where $\phi_{j}(\xi, t)$ and $a_{j}$ represents the modes and expansion coefficients while $\xi$ denotes the spatial vector. The modes computed from Eq. 9 are functions of space and time/frequency. Further splitting of space and time would lead to the need of computing only spatial modes. This can be achieved by separating the variables in the Eq. 9 assuming it is appropriate i.e. it can be presented as a linear combination of modes and their respective temporal coefficients, leading to the following expression

$$
\mathbf{x}(\xi, t)=\mathbf{q}(\xi, t)-\overline{\mathbf{q}}(\xi)=\sum_{j} \mathbf{a}_{j}(t) \phi_{j}(\xi)
$$

The snapshots of the unsteady component of scalar field $q(\xi, t)$ stacked into a matrix $\mathbf{X}$ for $m$ discrete time instances are used as input for the POD. $\mathbf{X}$ is a $n \times m$ matrix with $n$ as number of grid points (spatial data) and $m$ as number of snapshots (temporal data).

$$
\mathbf{X}=\left[\mathbf{x}\left(\xi, \mathbf{t}_{1}\right) \mathbf{x}\left(\xi, \mathbf{t}_{\mathbf{2}}\right) \ldots \mathbf{x}\left(\xi, \mathbf{t}_{\mathbf{m}}\right)\right]
$$

The optimal basis functions that can best represent the data described by $q(\xi, t)$ in a minimal number of modes $\phi_{j}(\xi)$ are then computed. This can either be done by computing eigenvectors $\phi_{j}$ and eigenvalues $\lambda_{j}$ (Eq. 12) from the covariance matrix $\mathbf{R}$ of $\mathbf{x}(\xi, t)$ which is a $n \times n$ matrix or by directly decomposing matrix $\mathbf{X}$ with Singular Value Decomposition (SVD) as 


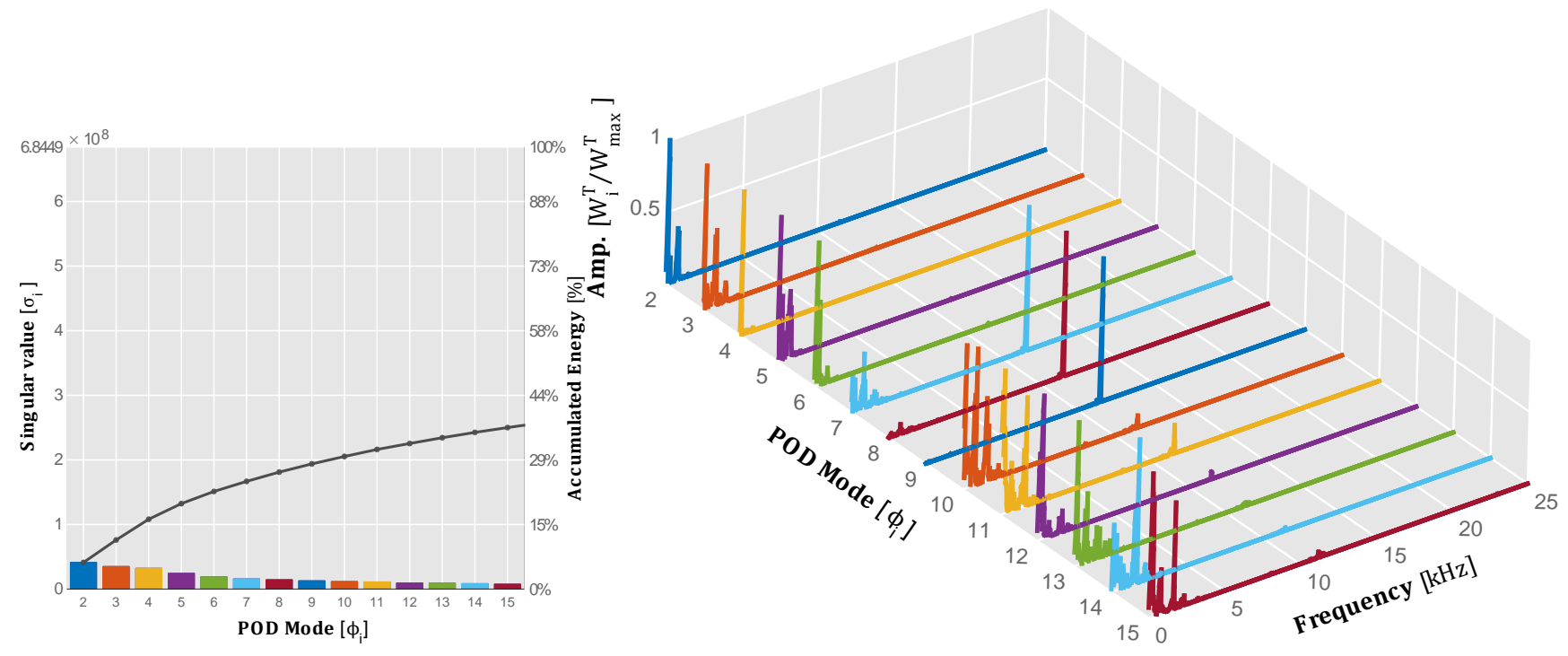

Figure 17: Energy levels $\lambda_{j}$ along with the normalised spectral content for the first 14 POD modes $\left(\phi_{2}\right.$ to $\left.\phi_{15}\right)$. Energy levels are arranged by relative energy content while the spectra of each mode is normalised by the maximum amplitude observed in that mode.

represented in Equation 14.

$$
\mathbf{R} \phi_{\mathbf{j}}=\lambda_{\mathbf{j}} \phi_{\mathbf{j}}
$$

where $\mathrm{R}$ is the covariance matrix

$$
\mathbf{R}=\sum_{i=1}^{m} \mathbf{x}\left(t_{i}\right) \mathbf{x}^{T}\left(t_{i}\right)=\mathbf{X} \mathbf{X}^{\mathbf{T}}
$$

$$
\mathbf{X}=\boldsymbol{\Phi} \Sigma \boldsymbol{\Psi}^{\mathbf{T}}
$$

where orthonormal matrices $\boldsymbol{\Phi}$ and $\boldsymbol{\Psi}$ are left and right singular vectors of $\mathbf{X}$ while diagonal matrix $\boldsymbol{\Sigma}$ contains singular values $\left(\sigma_{1} \sigma_{2} \ldots \sigma_{\mathbf{m}}\right)$.

The singular vectors $\boldsymbol{\Phi}$ and $\boldsymbol{\Psi}$ are corresponding eigenvectors of $\mathbf{X} \mathbf{X}^{\mathbf{T}}$ and $\mathbf{X}^{\mathbf{T}} \mathbf{X}$ respectively while singular values can be related to eigenvalues by $\sigma_{j}^{2}=\lambda_{j}$. The combination of $\boldsymbol{\Sigma} \boldsymbol{\Psi}^{\mathbf{T}}$

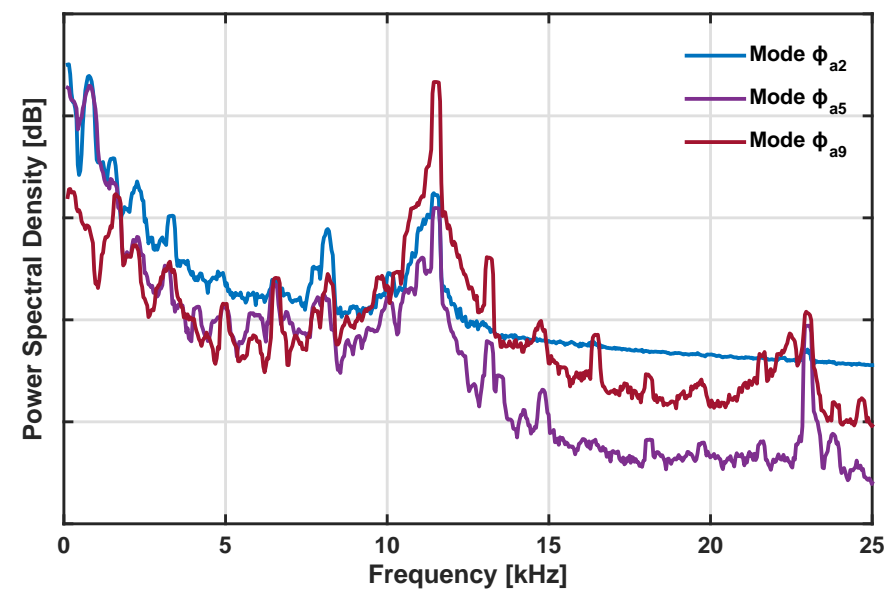

Figure 18: Spectral content of the time evolution $a_{i}(t)$ of POD modes $\Phi_{2,5,9}$, selected in order to analyse low-mid frequency range $\left(\boldsymbol{\Phi}_{2}\right)$, high frequency $\left(\boldsymbol{\Phi}_{9}\right)$ and overall $\left(\boldsymbol{\Phi}_{5}\right)$ frequency content of the total flow field. represent the time evolution $a_{j}(t)$ term for corresponding $\phi_{j}$. Therefore, SVD of $\mathbf{X}$ can directly yield POD modes $\boldsymbol{\Phi}$. Also, the robust nature of SVD based method against round off errors [32] makes it a better candidate relative to classical POD method and hence, SVD is used in this study to compute the POD modes. The output of POD would include the set of orthogonal modes $\phi_{j}(\xi)$ and their respective temporal coefficients $a_{j}(t)$ along with energy levels $\lambda_{j}$ arranged by relative energy content.

Since the matrix is of the order of grid points $(n \times m)$, a method similar to the method of snapshots [31] is used to make the SVD problem computationally tractable. The property of POD implying that decomposition using the data from a subset of the system instead of the entire system would yield the same dominant spatial modes is exploited in this work and SVD in reduced or economy sized is solved for a set of snapshots $\mathbf{x}\left(\xi_{i}, t\right)$ such that $i=1,2 \ldots r$ with $r \ll n$.

Applied to current compressor simulations, unsteady pressure scalar is the decomposed flow variable with the grid pressure values recorded for approximately 42 revolutions at a constant sampling time corresponding to $4 \mathrm{deg}$ impeller rotation. The pressure snapshots matrix $\mathbf{x}\left(\xi_{i}, t\right)$ is built using $10^{6}$ rows (r) by randomly selecting from the available $10^{7}$ (n) cells. The consistency in the pressure trace of the snapshot matrix is warranted by fixing the spatial vector $\xi_{i}$ in the pressure scalar $\mathbf{x}\left(\xi_{i}, t\right)$ i.e. each column of matrix $\mathbf{X}$ should represent the same cell. This is achieved by the selecting reference $10^{6}$ cells and then searching those reference cells across all snapshots. As the cells inside the rotating domain (impeller) changes their position due to sliding mesh, they are not considered at this stage. The pressure snapshot matrix is then decomposed by economy SVD to yield modes and respective temporal coefficients.

The energy levels for the first 14 modes $\left(\phi_{2}\right.$ to $\left.\phi_{15}\right)$ are presented in the form of a Pareto chart in Figure 17. First mode $\phi_{1}$ describes the time averaged pressure field and often displays 
alternating values in magnitude, implying the pressure oscillations. The energy is evenly distributed among the modes without any particularly dominant modes for the investigated near surge point. The normalised spectral features of these POD modes computed from their respective temporal coefficients $a_{j}(t)$ are also presented in Figure 17. The presence of expected lower frequency content in the spectra of these modes can be clearly observed. Three modes $\phi_{2}, \phi_{5}$ and $\phi_{9}$ are selected by assessing the normalised spectra for further investigation.

The detailed Power Spectral Density (PSD) spectra of these three modes $\phi_{2}, \phi_{5}$ and $\phi_{9}$ are shown in Figure 18. It can be seen that these modes provides a distinction in the features seen in the overall spectra with $\phi_{2}$ showing dominant content in the frequency range prior to first BPF while $\phi_{9}$ presents the content in the range after the first BPF. $\phi_{5}$ presents the content present mildly all across the spectrum. Although BPF can be seen in the spectra of all three modes but the strength of the BPF varies; least for $\phi_{2}$ and highest for $\phi_{9}$. The spatial distribution of these three modes is shown in Figure 19. Mode $\phi_{2}$ has the highest energy and corresponds to tonal characteristics including first and third rotating tone.

The spatial distribution of the pressure field for $\phi_{2}$ (top plot in Fig. 19) can be analysed to throw light on the source of the acoustic phenomenon. The positive and negative parts of the mode amplitude shown in the view of the whole domain indicates how the low-mid frequency range broadband content is propagating through the domain. Large fluctuating amplitudes are seen in the impeller downstream region specifically in the diffuser-volute region while the impeller upstream region presents a consistent pressure field. Further insight into the sources can be gained by plotting iso-volumes of only the highest energy areas of the mode. This has been realised in the detail view of the Fig. 19 by only displaying the $20^{\text {th }}$ and $80^{\text {th }}$ percentiles of the mode amplitude $\phi_{2}$ as the regions will alternatively hold the highest and the lowest $20 \%$ of the mode energy.

From the detailed view for $\phi_{2}$, it can be clearly seen that the sources of low-mid frequency broadband noises for the modelled operating condition are located in the impeller downstream components, suggestively diffuser-volute region. The impeller upstream regions, including the ported shroud do not show any high energy content of the $\phi_{2}$. It appears that, even though the PS is recirculating the low momentum flow from blade passages to the impeller inlet, the behaviour of recirculated flow does not have a significant impact on the generation or transmission of the mid-low frequency characteristics.

Mode $\phi_{9}$ corresponds to the tonal characteristics of the compressor noise spectrum including BPF and 'buzz-saw' tones. Spatial distribution of the pressure field (bottom plot in Fig. 19) indicates that the tonal noise propagate through the domain in the form of spiral structures that attenuates rapidly with the distance from impeller. A detailed view of the high energy structures indicates the rotating structures associated with the leading and trailing edges of the blade in the PS and diffuser region respectively as the sources of tonal noise. The PS cavity is seen to propagate the rotor alone pressure field causing 'buzz-saw' tones in the impeller upstream direction.

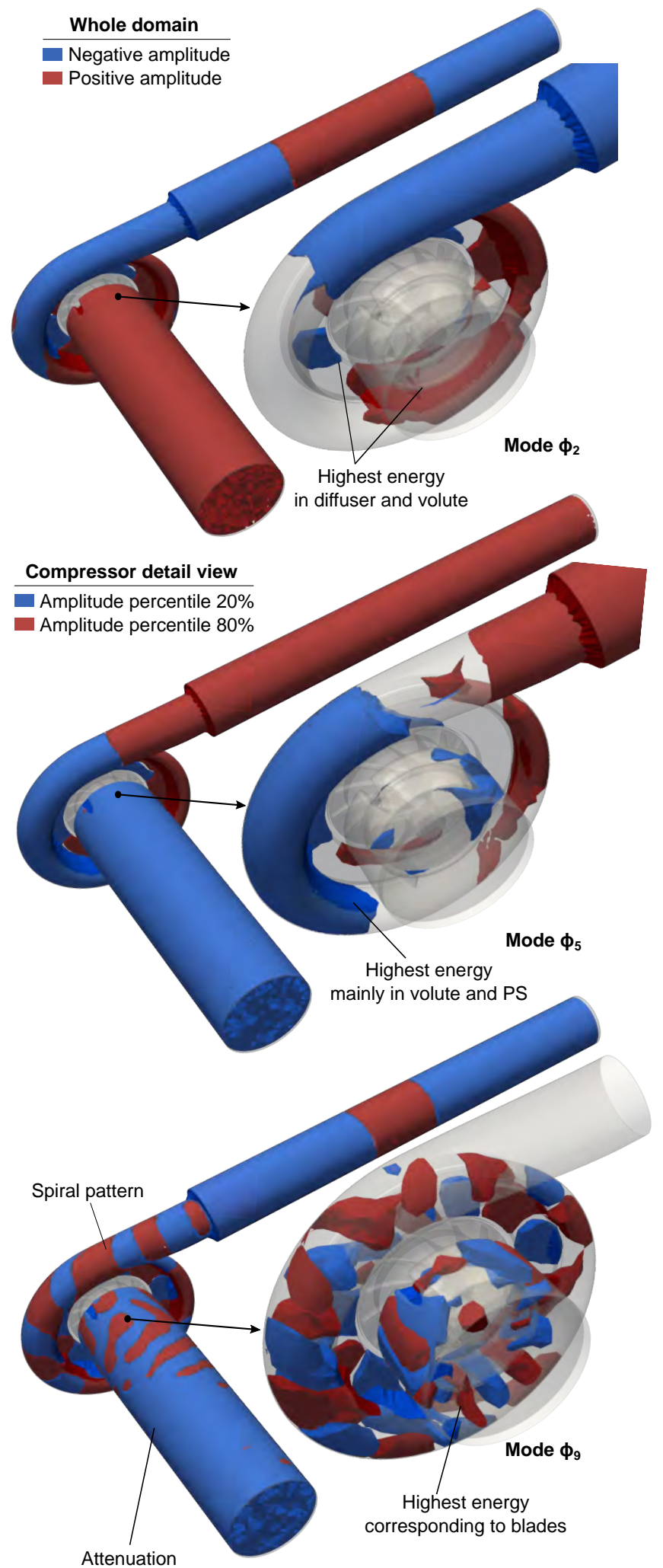

Figure 19: Spatial distribution of POD modes $\boldsymbol{\Phi}_{2,5,9}$, visualized by isosurfaces of positive and negative values in the whole domain, and by isovolumes of top $20 \%$ energy (percentiles $20 \%$ and $80 \%$ ) in the compressor detail view. 
Mode $\phi_{5}$ corresponds to the amalgamation of the low and high frequency characteristics showing some part of broadband noise as well as mild BPF and 'buzz-saw' tones. Spatial distribution of the pressure field (middle plot in Fig. 19) is similar to that of $\phi_{2}$ along with small mode features in the PS cavity region. The detailed view shows that the high-energy structures are concentrated in the volute and PS cavity region reinforcing that the broadband content is localised in the impeller downstream region while tonal content is propagated through both diffuser and PS cavity regions.

\section{Conclusions}

In this investigation, the acoustic characteristics of a commercial turbocharger compressor equipped with a ported shroud operating in soft surge region have been studied through a numerical simulation of the flow field. A range of turbulence models and temporal discretisation have been explored but the results are briefly presented with an intent to showcase their impact on performance and acoustic predictions. Boundary conditions were obtained through experimental testing of the selected turbocharger, also acquiring data that is used to validate the numerical model performance, demonstrating a deviation of around $1 \%$ with respect to measurements. The acoustic predictions of the numerical model are validated by comparing them with experimentally measured spectra for the position near impeller and diffuser. The numerical and experimental spectra are seen to be in a reasonable agreement although numerical model could not predict the broadband noise seen after the BPF in the experimental spectra.

Acoustic signature of the near impeller probe predicted by the model shows BPF and weak rotor shaft speed ('buzz saw') harmonics. Similar features are seen in the spectra of middiffuser probes. The 'mid-tones' between 'buzz-saw' tones that are seen in the spectra of design operation are absent in the near surge operation. It is also interesting to note that the four diffuser probes located $90^{\circ}$ apart shows similar characteristics implying the low impact of the asymmetric flow seen in the diffuser for near surge operation. Propagation of the acoustic phenomena through the inlet and outlet ducts is computed from the decomposed pressure wave using beamforming and $\mathrm{MoC}$ to enhance the spectral content. The numerical and experimental duct spectra obtained from the decomposed pressure wave using beamforming showed good agreement. In contrast, the correlation between the experimental duct spectra computed from the raw pressure signal and the numerical results is unsatisfactory but to the presence of higher order duct modes. Tonal noise can be identified in the numerical spectra of the ducts but the broadband noise corresponding to 'whoosh' noise is not seen for the studied operating condition. Broadband noise in the range of $12-14 \mathrm{kHz}$ is identified in the measured spectra of the outlet duct. This broadband can be loosely related to so called TCN but it is observed after the first BPF, in contrast to what is usually seen in literature.

Analysis of the flow characteristics was also performed in order to characterise the near surge operation along with the particularities introduced by the use of the PS. Results demonstrated a unidirectional radially outward flow at the PS slot in contrast to the push and pull behaviour seen for the design operation. The flow then exits the PS cavity in the form of high velocity jets that interacts with the incoming flow leading to the propagation of unsteadiness upstream. Low velocity stall cells and their circumferential propagation along the impeller blades are also identified. To investigate the acoustic sources, Proper Orthogonal Decomposition was applied to the numerical simulation results. The decomposition of the total fluid field (and therefore, its spectral signature) into its constituent modes allowed the separate analysis of different frequency characteristics of the compressor pressure field. Inspection of the spatial distribution of the modal energy illustrated propagation patterns and, more importantly, the role of the impeller downstream components i.e. diffuser and volute in housing the more energetic sources of the broadband content. Ported shroud cavity is not seen to be associated with the significant source of noise content, it does play a small role in the propagation of the tonal content in the impeller upstream direction.

In conclusion, various acoustic features and their respective spatial pressure distribution are identified along with the unsteadiness in the compressor. It is thus recommended that further numerical post processing and experimental studies should be conducted to identify the flow mechanisms of the individual frequency peaks in order to ensure that the optimal performance and noise characteristics of the ported shroud compressors.

\section{Acknowledgements}

The project is sponsored and supported by BorgWarner Turbo Systems and the Regional Growth Fund (RGF Grant Award 01.09.07.01/1789C). The authors would like to thank BorgWarner Turbo Systems for permission to publish the results presented in this paper. The support of the HPC group at the University of Huddersfield is gratefully acknowledged.

\section{References}

[1] E. M. Greitzer, The stability of pumping systems - the 1980 freeman scholar lecture, Journal of Fluids Engineering 103 (2) (1981) 193-242. doi: 10.1115/1.3241725.

[2] F. Fisher, Application of map width enhancement devices to turbocharger compressor stages, Tech. rep., SAE Technical Paper (1988). doi:10. $4271 / 880794$.

[3] I. Macdougal, R. Elder, The improvement of operating range in a small, high speed, centrifugal compressor using casing treatment, in: IMechE Conference on Turbochargers and Turbocharging, Paper, no. C32/82, 1982.

[4] C. Teng, S. Homco, Investigation of compressor whoosh noise in automotive turbochargers, SAE International Journal of Passenger CarsMechanical Systems 2 (2009-01-2053) (2009) 1345-1351. doi:10. 4271/2009-01-2053.

[5] D. Evans, A. Ward, Minimising turbocharger whoosh noise for diesel powertrains, Tech. rep., SAE Technical Paper (2005). doi:10.4271/ 2005-01-2485.

[6] G. Gaude, T. Lefevre, R. Tanna, K. Jin, T. McKitterick, S. Armenio, Experimental and computational challenges in the quantification of turbocharger vibro-acoustic sources, in: INTER-NOISE and NOISE-CON Congress and Conference Proceedings, Vol. 2008, Institute of Noise Control Engineering, 2008, pp. 5754-5767. 
[7] E. P. Trochon, A new type of silencers for turbocharger noise control, Tech. rep., SAE Technical Paper (2001). doi:10.4271/ 2001-01-1436.

[8] N. Figurella, R. Dehmer, A. Selamet, K. Tallio, K. Miazgowicz, R. Wade, A. Karim, P. Keller, J. Shutty, Effect of inlet vanes on centrifugal compressor acoustics and performance, Noise Control Engineering Journal 62 (4) (2014) 232-237. doi : 10.3397/1/376223.

[9] A. Torregrosa, A. Broatch, X. Margot, J. García-Tíscar, Y. Narvekar, R. Cheung, Local flow measurements in a turbocharger compressor inlet, Experimental Thermal and Fluid Sciencedoi:10.1016/j. expthermflusci.2017.07.007.

[10] N. Figurella, R. Dehner, A. Selamet, K. Tallio, K. Miazgowicz, R. Wade, Noise at the mid to high flow range of a turbocharger compressor, Noise Control Engineering Journal 62 (5) (2014) 306-312. doi:10.3397/1/ 376229.

[11] A. Broatch, J. Galindo, R. Navarro, J. García-Tíscar, A. Daglish, R. Sharma, Simulations and measurements of automotive turbocharger compressor whoosh noise, Engineering Applications of Computational Fluid Mechanics 9 (1) (2015) 12-20. doi : 10.1080/19942060 . 2015. 1004788.

[12] T. Raitor, W. Neise, Sound generation in centrifugal compressors, Journal of Sound and Vibration 314 (3) (2008) 738-756. doi : 10.1016/j · jsv . 2008.01.034.

[13] F. Mendonça, O. Baris, G. Capon, Simulation of radial compressor aeroacoustics using CFD, in: Proceedings of ASME Turbo Expo, 2012, pp. 1823-1832. doi:10.1115/GT2012-70028.

[14] A. Broatch, J. Galindo, R. Navarro, J. García-Tíscar, Methodology for experimental validation of a CFD model for predicting noise generation in centrifugal compressors, International Journal of Heat and Fluid Flow 50 (2014) 134-144. doi:10.1016/j.ijheatfluidflow. 2014.06.006.

[15] B. Semlitsch, M. Mihăescu, Flow phenomena leading to surge in a centrifugal compressor, Energy 103 (2016) 572-587. doi:10.1016/j. energy.2016.03.032

[16] E. Sundström, B. Semlitsch, M. Mihaescu, Centrifugal compressor: The sound of surge, in: Proceedings of the 21st AIAA/CEAS Aeroacoustics Conference, Dallas, TX, 2015. doi:10.2514/6.2015-2674.

[17] V. Jyothishkumar, M. Mihaescu, B. Semlitsch, L. Fuchs, Numerical flow analysis in a centrifugal compressor near surge condition, AIAA20132730doi: $10.2514 / 6.2013-2730$.

[18] A. Torregrosa, A. Broatch, X. Margot, J. García-Tíscar, Experimental methodology for turbocompressor in-duct noise evaluation based on beamforming wave decomposition, Journal of Sound and Vibration 376 (2016) 60-71. doi : 10.1016/j.jsv . 2016.04.035.

[19] A. CFX-Solver, Theory guide, Release 11.

[20] F. Nicoud, F. Ducros, Subgrid-scale stress modelling based on the square of the velocity gradient tensor, Flow, Turbulence and Combustion 62 (3) (1999) 183-200. doi:10.1023/A :1009995426001. URL https : //doi .org/10.1023/A: 1009995426001

[21] F. Menter, M. Kuntz, Development and application of a zonal des turbulence model for CFX-5, Ansys, CFX-Validation Report, Technical Report No. CFX-VAL17/0503.

[22] F. R. Menter, Stress-blended eddy simulation (SBES) - a new paradigm in hybrid rans-les modeling, in: Symposium on Hybrid RANS-LES Methods, no. 6, 2016.

[23] A. Torregrosa, P. Fajardo, A. Gil, R. Navarro, Development of nonreflecting boundary condition for application in 3D computational fluid dynamics codes, Engineering Applications of Computational Fluid Mechanics 6 (3) (2012) 447-460. doi:10.1080/19942060.2012. 11015434.

[24] I. ANSYS, I. ANSYS, CFD help manual, ANSYS, Inc., Canonsburg, PA

[25] P. Chow, M. Cross, K. Pericleous, A natural extension of the conventional finite volume method into polygonal unstructured meshes for CFD application, Applied Mathematical Modelling 20 (2) (1996) 170-183. doi : 10.1016/0307-904X (95) 00156-E.

[26] M. Tritthart, D. Gutknecht, Three-dimensional simulation of free-surface flows using polyhedral finite volumes, Engineering Applications of Computational Fluid Mechanics 1 (1) (2007) 1-14. doi:10.1080/ 19942060.2007.11015177.

[27] Z. Sun, C. Tan, D. Zhang, Flow field structures of the impeller backside cavity and its influences on the centrifugal compressor, in: Proceedings of ASME TurboExpo, 2009, pp. 8-12. doi : 10.1115/GT2009-59879.
[28] S. Sharma, A. Jupp, Broatch, J. Nickson, García-Tíscar, A. K. Nickson, J. M. Allport, Acoustic characteristics of a ported shroud turbocompressor operating at design condition, International Journal of Engine Research, Accepted.doi : 10.1177/1468087418814635.

[29] J. G. Tíscar, Experiments on turbocharger compressor acoustics, Ph.D. thesis, Polytechnic University of Valencia. Department of Thermal Machines and Motors - Department of Heat Engines and Machines (2017). doi:10.4995/Thesis/10251/79552.

[30] S. Sharma, M. L. Jupp, A. K. Nickson, J. M. Allport, Ported shroud flow processes and their effect on turbocharger compressor operation, in: ASME Turbo Expo 2017: Turbomachinery Technical Conference and Exposition, American Society of Mechanical Engineers, 2017, pp. V02CT44A017-V02CT44A017. doi:10.1115/GT2017-63678.

[31] K. Taira, S. Brunton, S. Dawson, C. Rowley, T. Colonius, B. J. McKeon, O. Schmidt, S. Gordeyev, V. Theofilis, L. Ukeiley, Modal analysis of fluid flows: An overview 55. doi:https://doi.org/10.2514/1. J056060. 